\title{
Reduced Basis ANOVA Methods for Partial Differential Equations with High-Dimensional Random Inputs
}

\author{
Qifeng Liao ${ }^{\mathrm{a}}$, Guang Lin ${ }^{\mathrm{b}, *}$ \\ ${ }^{a}$ School of Information Science and Technology, ShanghaiTech University, Shanghai 200031, China \\ ${ }^{b}$ Department of Mathematics, Purdue University, West Lafayette, Indiana 47907, USA
}

\begin{abstract}
In this paper we present a reduced basis ANOVA approach for partial deferential equations (PDEs) with random inputs. The ANOVA method combined with stochastic collocation methods provide model reduction in high-dimensional parameter space through decomposing high-dimensional inputs into unions of low-dimensional inputs. In this work, to further reduce the computational cost, we investigate spatial low-rank structures in the ANOVA-collocation method, and develop efficient spatial model reduction techniques using hierarchically generated reduced bases. We present a general mathematical framework of the methodology, validate its accuracy and demonstrate its efficiency with numerical experiments.
\end{abstract}

Keywords: adaptive ANOVA, stochastic collocation, reduced basis methods, uncertainty quantification.

\section{Introduction}

Over the past few decades there has been a rapid development in numerical methods for solving partial differential equations (PDEs) with random inputs. This explosion in interest has been driven by the need of conducting uncertainty quantifica5 tion for practical problems. In particular, uncertainty quantification for problems with high-dimensional random inputs gains a lot of interest. High-dimensional inputs exist

\footnotetext{
${ }^{*}$ Corresponding author

Email addresses: liaoqf@shanghaitech.edu.cn (Qifeng Liao), guanglin@purdue.edu (Guang
} Lin) 
in many practical problems, for example, problems with inputs described by random processes with short correlation lengths. This paper is devoted to high-dimensional uncertainty quantification problems.

To the authors' knowledge, there exist two main kinds of computational challenges for efficiently solving these high-dimensional uncertainty quantification problems in the context of PDEs: curse of dimensionality for the parameter space, and large-rank structures in spatial approximations. The curse of dimensionality is an obstacle to apply stochastic spectral methods 1 1, 2, 3, 4, 5]. As discussed in our earlier study [6], highdimensional random inputs can also lead to large spatial ranks, which make it difficult to apply model reduction techniques for spatial approximations.

Many new methods are developed to resolve these challenging high-dimensional and large-rank problems. For parameter space discretization, ANOVA methods [7, 8, 9, 10, 11, 12, 13, 14, 15] are developed to decompose a high-dimensional parameter space

20 into a union of low-dimensional spaces, such that stochastic collocation methods can then be efficiently applied. Besides ANOVA, adaptive sparse grids $[16,3,17,18,19$, 20], multi-element collocation [21] and compressive sensing methods [22, 23, 24] are also developed to discretize high-dimensional parameter spaces. For efficient spatial approximation, localized reduced basis methods are developed to resolve large-rank problems, for example, model reduction based on spitting parameter domains [25, 26] and that based on spatial domain decomposition methods [27, 28, 29]. In addition, efficient decomposition methods for both parameter and spatial spaces are developed in [30, 31, 32], and general distributed uncertainty quantification approaches are proposed in $[33,34,35,36]$.

We in this paper focus on the ANOVA decomposition method. We note that lowdimensional parameter spaces generated in the ANOVA decomposition [12, 14] can also lead to low-rank structures in spatial approximations. To capture these low-rank spatial structures, we develop a hierarchical reduced basis method. Since these lowrank structures give very small sizes of reduced bases, our proposed method can significantly improve the computational efficiency of the ANOVA method. In addition, we remark that using model reduction methods to enhance the performance of stochastic spectral methods are also investigated in [37, 6, 38, 39]. 
An outline of the paper is as follows. We present our problem setting and review the ANOVA-collocation combination in the next section. In Section 3, we review the reduced basis methods for parameterized PDEs. Our main algorithm is presented in Section 4 Numerical results are discussed in Section 5. Second 6 concludes the paper.

\section{Problem setting and ANOVA decomposition}

Let $D \subset \mathbb{R}^{d}(d=2,3)$ denote a spatial domain which is bounded, connected and with a polygonal boundary $\partial D$, and $x \in \mathbb{R}^{d}$ denote a spatial variable. Let $\xi$ be a vector which collects a finite number of random variables. The dimension of $\xi$ is denoted by $M$, i.e., we write $\xi=\left[\xi_{1}, \ldots, \xi_{M}\right]^{T}$. The probability density function of $\xi$ is denoted by $\pi(\xi)$. In this paper, we restrict our attention to the situation that $\xi$ has a bounded and connected support. We next assume the support of $\xi$ to be $I^{M}$ where $I:=[-1,1]$, since any bounded connected domain in $\mathbb{R}^{M}$ can be mapped to $I^{M}$. The physics of problems considered in this paper are governed by a PDE over the spatial domain $D$ and boundary conditions on the boundary $\partial D$. The global problem solves the governing equations which are stated as: find $u(x, \xi): D \times I^{M} \rightarrow \mathbb{R}$, such that

$$
\begin{aligned}
\mathcal{L}(x, \xi ; u(x, \xi))=f(x) & \forall(x, \xi) \in D \times I^{M}, \\
\mathfrak{b}(x, \xi ; u(x, \xi))=g(x) & \forall(x, \xi) \in \partial D \times I^{M},
\end{aligned}
$$

where $\mathcal{L}$ is a partial differential operator and $\mathfrak{b}$ is a boundary operator, both of which can have random coefficients. $f$ is the source function and $g$ specifies the boundary 45 conditions. In the rest of this section, we review the ANOVA decomposition [20, 14] and stochastic collocation methods [3].

\subsection{ANOVA decomposition}

Following the presentation in [11], we first introduce notation for indices. In general, any subset of $\{1, \ldots, M\}$ denotes an index. For an index $t \subseteq\{1, \ldots, M\},|t|$ denotes the cardinality of $t$. For the special case that $t=\emptyset$, we define $|t|=0$. For an index $t \neq \emptyset$, we sort its elements in ascending order and express it as $t=\left(t_{1}, \ldots, t_{|t|}\right)$ with $t_{1}<t_{2} \ldots<t_{|t|}$. In addition, we also call $|t|$ the (ANOVA) order of $t$, and call $t$ a $|t|$-th 
order index. For a given ANOVA order $i=0, \ldots, M$, we define the following index sets

$$
\begin{aligned}
\mathfrak{I}_{i} & :=\{t|t \subset\{1, \ldots, M\},| t \mid=i\}, \\
\mathfrak{I}_{i}^{\star} & :=\cup_{j=0,1, \cdots, i} \mathfrak{I}_{j}, \\
\mathfrak{I} & :=\mathfrak{I}_{M}^{\star}=\cup_{j=0,1, \cdots, M} \mathfrak{I}_{j} .
\end{aligned}
$$

55 The sizes of the above sets (numbers of elements that they contain) are denoted by $\left|\mathfrak{I}_{i}\right|$, $\left|\mathfrak{I}_{i}^{\star}\right|$ and $|\mathfrak{I}|$ respectively. From the above definition, $\mathfrak{I}_{0}=\{\emptyset\}$ and $\left|\mathfrak{I}_{0}\right|=1$ (since $\{\emptyset\}$ is not empty). For a given index $t=\left(t_{1}, \ldots, t_{|t|}\right) \in \mathfrak{I}$ with $|t|>0, \xi_{t}$ denotes a random vector collecting components of $\xi$ associated with $t$, i.e., $\xi_{t}:=\left[\xi_{t_{1}}, \ldots, \xi_{t_{\mid l}}\right]^{T} \in I^{|t|}$, and we denote the probability density function of $\xi_{t}$ by $\pi_{t}$.

While ANOVA methods for solving stochastic PDEs are discussed in detail in «12, 14], we in this paper only focus on the anchored ANOVA method [40]. Given an anchor point $c=\left[c_{1}, \ldots, c_{M}\right]^{T} \in I^{M}$, the anchored ANOVA method decomposes the solution $u(x, \xi)$ of the global problem (1)-(2) as follows

$$
\begin{aligned}
u(x, \xi) & =u_{0}(x)+u_{1}\left(x, \xi_{1}\right)+\ldots+u_{1,2}\left(x, \xi_{1,2}\right)+\ldots \\
& =\sum_{t \in \mathfrak{I}} u_{t}\left(x, \xi_{t}\right),
\end{aligned}
$$

where we denote $u_{\emptyset}\left(x, \xi_{\emptyset}\right):=u_{0}(x)$ for convenience, and each term in (3) is specified 65 as

$$
\begin{aligned}
u_{\emptyset}\left(x, \xi_{\emptyset}\right) & :=u_{0}(x):=u(x, c), \\
u_{t}\left(x, \xi_{t}\right) & :=u\left(x, c, \xi_{t}\right)-\sum_{s \subset t} u_{s}\left(x, \xi_{s}\right) .
\end{aligned}
$$

In (4), $u(x, c)$ is the solution of the deterministic version of (10)-(2) with the realization $\xi=c$, while $u\left(x, c, \xi_{t}\right)$ in (5) is the solution of a semi-deterministic version of (1)-(2) through fixing $\xi_{i}=c_{i}$ for $i \in\{1, \ldots, M\} \backslash t$, i.e.,

$$
u\left(x, c, \xi_{t}\right):=u\left(x, \xi^{c, t}\right),
$$

where $\xi^{c, t}:=\left[\xi_{1}^{c, t}, \ldots, \xi_{M}^{c, t}\right]^{T} \in I^{M}$ is defined through

$$
\xi_{i}^{c, t}:=\left\{\begin{array}{ll}
c_{i} & \text { for } i \in\{1, \ldots, M\} \backslash t \\
\xi_{i} & \text { for } i \in t
\end{array} .\right.
$$


From above, it is clear that for any $t \in \mathfrak{I}$ with $|t|>0, u\left(x, c, \xi_{t}\right)$ maps $D \times I^{|t|}$ to $\mathbb{R}$ and satisfies

$$
\begin{aligned}
\mathcal{L}_{t}\left(x, \xi_{t} ; u\left(x, c, \xi_{t}\right)\right)=f(x) & \forall\left(x, \xi_{t}\right) \in D \times I^{|t|}, \\
\mathfrak{b}_{t}\left(x, \xi_{t} ; u\left(x, c, \xi_{t}\right)\right)=g(x) & \forall\left(x, \xi_{t}\right) \in \partial D \times I^{|t|},
\end{aligned}
$$

70

where $\mathcal{L}_{t}$ and $\mathrm{b}_{t}$ are defined through putting (6) into (1)-(2). We refer to (7)-(8) as a (parametrically) $|t|$-dimensional local problem, while the global problem is (1)-(2).

Note that for a given positive integer $i \leq M$, there are $\left(\begin{array}{c}M \\ i\end{array}\right)$ ANOVA terms at $i$-th order, i.e., $\left|\mathfrak{I}_{i}\right|=\left(\begin{array}{c}M \\ i\end{array}\right)$. When $M$ is large, it can be a very large number even for a relative small expansion order, e.g., $i=2$. So, the total number of ANOVA terms $(|\mathfrak{I}|)$ in (3) can be large, and computing them can be expensive. Especially, computing each high order term is already very expensive. For this purpose, we would recall the motivation of using ANOVA decomposition—only part of low order terms in the ANOVA expansion are expected to be active based on some selection criteria, which gives the opportunity to build an adaptive ANOVA expansion with these active low order terms as an efficient surrogate to approximate the exact solution $u(x, \xi)$ (see [12, 14]).

We denote the sets consisting of selected indices at each order by $\mathcal{J}_{i} \subseteq \mathfrak{I}_{i}$ for $i=0, \ldots, M$ (details of constructing these sets will be discussed next). Similarly to the definitions of $\mathfrak{I}_{i}^{\star}$ and $\mathfrak{I}$, we define $\mathcal{J}_{i}^{\star}:=\cup_{j=0, \ldots, i} \mathcal{J}_{j}$ and $\mathcal{J}:=\mathcal{J}_{M}^{\star}$. With selected (active) indices, the solution $u(x, \xi)$ of (1)-(2) can be approximated by

$$
u(x, \xi) \approx u_{\mathcal{J}}(x, \xi):=\sum_{t \in \mathcal{J}} u_{t}\left(x, \xi_{t}\right),
$$

where $u_{t}$ is defined in (5). As discussed in [12, 14], several popular criteria to select active terms (or indices) are discussed, e.g., using relative mean values and relative variance values. For simplicity, we use relative mean values to select indices. For a given term $u_{t}$ in (9) with $t \in \mathcal{J}$ and $|t|>0$, its relative mean value is defined by

$$
\gamma_{t}:=\frac{\left\|\mathbf{E}\left(u_{t}\right)\right\|_{0, D}}{\left\|\sum_{s \in \mathcal{J}_{|t|-1}^{\star}} \mathbf{E}\left(u_{s}\right)\right\|_{0, D}},
$$

where $\|\cdot\|_{0, D}$ denotes the $L^{2}$ function norm, and $\mathbf{E}\left(u_{t}\right)$ denotes the mean function of $u_{t}$

$$
\mathbf{E}\left(u_{t}\left(x, \xi_{t}\right)\right):=\int_{I^{|t|}} u_{t}\left(x, \xi_{t}\right) \pi_{t}\left(\xi_{t}\right) d \xi_{t} .
$$


Supposing $\mathcal{J}_{i}$ is given for an order $i \leq M-1, \mathcal{J}_{i+1}$ is constructed through the following two steps presented in [12]. First, active terms in $\mathcal{J}_{i}$ needs to be selectedthat is to construct a set $\tilde{\mathcal{J}}_{i}:=\left\{t \mid t \in \mathcal{J}_{i}\right.$ and $\gamma_{t} \geq t_{\text {ol }}$ anova $\}$, where tol $_{\text {anova }}$ is a given tolerance. After that, the index set of the next order $\mathcal{J}_{i+1}$, is constructed by

$$
\mathcal{J}_{i+1}:=\left\{t \mid t \in \mathfrak{I}_{i+1} \text {, and any } s \subset t \text { with }|s|=i \text { satisfies } s \in \tilde{\mathcal{J}}_{i}\right\} .
$$

To start this constructing procedure, we set $\mathcal{J}_{0}=\mathfrak{I}_{0}=\emptyset$ and $\mathcal{J}_{1}=\mathfrak{I}_{1}=\{1, \ldots, M\}$. From the studies in $[12,14]$, the size of $\mathcal{J}$ is typically much smaller than that of $\mathfrak{I}$, and $\mathcal{J}$ typically only contains low order terms.

\subsection{Stochastic collocation}

As discussed above, in order to obtain each expansion term in the ANOVA approximation (9), we need to compute each $u\left(x, c, \xi_{t}\right)$ in (5) for $t \in \mathcal{J}$. When $|t|=0$, $u_{0}(x)=u_{\emptyset}\left(x, \xi_{\emptyset}\right)=u(x, c)$ is obtained through solving a deterministic version of (1)(2); when $|t|>0$, we need to solve local stochastic PDEs (7)-(8) to obtain $u\left(x, c, \xi_{t}\right)$. The stochastic collocation method is applied to construct an interpolation approximation of each $u\left(x, c, \xi_{t}\right)$ in [14]. Choosing proper interpolation points is curial for the collocation methods [3]. In this paper, we follow the tensor style Clenshaw-Curtis collocation used in [14]. For a given collocation sample set $\Theta_{t} \subset I^{|t|}$ (a set consisting of collocation points), the corresponding collocation approximation of $u\left(x, c, \xi_{t}\right)$ can be written as

$$
u^{\mathrm{sc}}\left(x, c, \xi_{t}\right):=\sum_{\xi_{t}^{(j)} \in \Theta_{t}} u\left(x, c, \xi_{t}^{(j)}\right) \Phi_{\xi_{t}^{(j)}}\left(\xi_{t}\right)
$$

where the collocation coefficients $\left\{u\left(x, c, \xi_{t}^{(j)}\right), \xi_{t}^{(j)} \in \Theta_{t}\right\}$ are deterministic solutions of (7)-(8) at collocation sample points, the superscript $j \in\left\{1 \ldots,\left|\Theta_{t}\right|\right\}$ denotes the

$j$-th collocation sample point, and $\left\{\Phi_{\xi_{t}^{(j)}}\left(\xi_{t}\right), \xi_{t}^{(j)} \in \Theta_{t}\right\}$ are interpolation polynomials [3]. Combining (5), (9) and (12), an ANOVA-collocation approximation (denoted by $\left.u_{\mathcal{J}}^{\mathrm{sc}}(x, \xi)\right)$ for the exact solution $u(x, \xi)$ is defined by

$$
\begin{aligned}
u_{\mathcal{J}}^{\mathrm{sc}}(x, \xi) & :=\sum_{t \in \mathcal{T}} u_{t}^{\mathrm{sc}}\left(x, \xi_{t}\right), \\
u_{t}^{\mathrm{sc}}\left(x, \xi_{t}\right) & :=u^{\mathrm{sc}}\left(x, c, \xi_{t}\right)-\sum_{s \subset t} u_{s}^{\mathrm{sc}}\left(\cdot, \xi_{s}\right),
\end{aligned}
$$


where we set $u_{\emptyset}^{\mathrm{sc}}\left(x, \xi_{\emptyset}\right):=u(x, c)$ for convenience.

As discussed in Section 2.1, we use the relative mean value (10) to select active indices. Based on this stochastic collocation formulation, the mean function of each $u_{t}^{\mathrm{sc}}$ in (13) can be approximated by the following quadrature

$$
\tilde{\mathbf{E}}\left(u_{t}^{\mathrm{sc}}\left(x, \xi_{t}\right)\right):=\sum_{\xi_{t}^{(j)} \in \Theta_{t}} u_{t}^{\mathrm{sc}}\left(x, \xi_{t}^{(j)}\right) \pi_{t}\left(\xi_{t}^{(j)}\right) w_{\xi_{t}^{(j)}},
$$

where $\left\{w_{\xi_{t}^{(j)}}, \xi_{t}^{(j)} \in \Theta_{t}\right\}$ are the weights of the Clenshaw-Curtis tensor quadrature [14]. Then the relative mean value (10) for each $t \in \mathcal{J}$ with $|t|>0$ can be approximated by

$$
\tilde{\gamma}_{t}:=\frac{\left\|\tilde{\mathbf{E}}\left(u_{t}^{\mathrm{sc}}\right)\right\|_{0, D}}{\left\|\sum_{s \in \mathcal{J}_{|| \mid-1}^{\star}} \tilde{\mathbf{E}}\left(u_{s}^{\mathrm{sc}}\right)\right\|_{0, D}} .
$$

\section{Spatial discretization and reduced basis approximation}

As introduced in Section 2.2 in order to construct the ANOVA-collocation approximation (13)-(14), solutions of the deterministic versions of (7)-(8) at collocation points (see (12) ) need to be computed. In this section, we discuss finite element and reduced basis approximations for deterministic PDEs.

To begin with, we state the finite element approximation of the deterministic version of each local problem (7)-8) corresponding to a given realization of $\xi_{t}$ as: given a finite element space $X^{h}$ with $N_{h}$ degrees of freedom, find $u_{h}\left(x, c, \xi_{t}\right) \in X^{h}$ such that

$$
\mathfrak{B}_{\xi_{t}}\left(u_{h}\left(x, c, \xi_{t}\right), v\right)=l(v), \quad \forall v \in X^{h} .
$$

As usual, a finite element solution $u_{h}$ is referred to as a snapshot. With the finite element approximation, we include the standard ANOVA approach in Algorithm 1 following the presentation in [12] for completeness, while Algorithm 11 can be considered as a summary of Section 2 In Algorithm $1, u_{h}(x, c)$ denotes the solution of (17) for $\xi_{t}=c$ (the snapshot of the global problem (11)-(2) at $\xi=c$ ).

Next, the reduced basis approximation is stated as: given a set of reduced basis functions $Q_{t}:=\left\{q_{t}^{(1)}, \cdots, q_{t}^{\left(N_{r}\right)}\right\} \subset X^{h}$, find $u_{r}\left(x, c, \xi_{t}\right) \in \operatorname{span}\left\{Q_{t}\right\}$ such that

$$
\mathfrak{B}_{\xi_{t}}\left(u_{r}\left(x, c, \xi_{t}\right), v\right)=l(v), \quad \forall v \in \operatorname{span}\left\{Q_{t}\right\}
$$




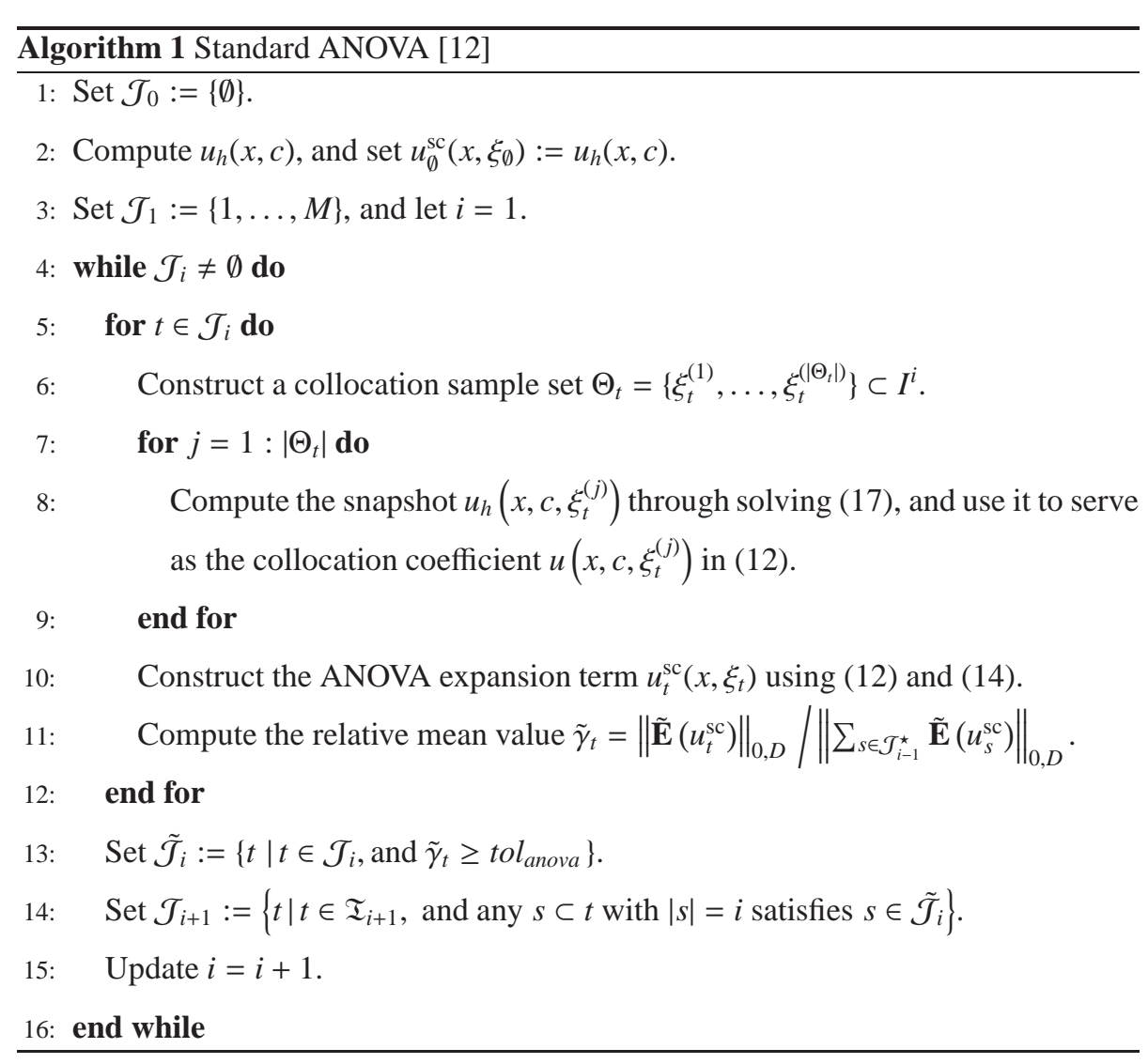


As discussed in [41], two conflicting requirements need to be balanced for the size of

the reduced basis: the size $N_{r}$ should be small such that it is cheap to solve the reduced problem (18), but $N_{r}$ needs to be large enough such that the reduced solution $u_{r}\left(x, c, \xi_{t}\right)$ approximates the finite element solution $u_{h}\left(x, c, \xi_{t}\right)$ well. Methods for generating the reduced basis $Q_{t}$ have been proposed in the literature. These methods can be broadly classified into the following two kinds (see [41, 42] for detailed reviews).

The first kind is proper orthogonal decomposition (POD) [43, 44, 45]. We here briefly review this type of methods following the presentation in [45]. For a given finite sample set $\Xi \subset I^{|t|}$ with size $|\Xi|$, a finite snapshot set is defined by

$$
S_{\Xi}^{t}:=\left\{u_{h}\left(x, c, \xi_{t}\right), \xi_{t} \in \Xi\right\}
$$

The matrix form of $S_{\Xi}^{t}$ is denoted by $\mathbf{S}_{\Xi}^{t} \in \mathbb{R}^{N_{h} \times|\Xi|}$, i.e., each column of $\mathbf{S}_{\Xi}^{t}$ is the vector of basis function coefficients of a finite element solution. Assuming $|\Xi|<N_{h}$, let $\mathbf{S}_{\Xi}^{t}=U \Sigma V^{T}$ denote the singular value decomposition (SVD) of $\mathbf{S}_{\Xi}^{t}$, where $U=$ $\left(\mathbf{q}_{1}, \cdots, \mathbf{q}_{|\Xi|}\right)$ and $\Sigma=\operatorname{diag}\left(\sigma_{1}, \cdots, \sigma_{|\Xi|}\right)$ with $\sigma_{1} \geq \sigma_{2} \geq \cdots \geq \sigma_{|\Xi|} \geq 0$. The basis $Q_{t}$ is then given by the first $k$ left singular vectors $\left(\mathbf{q}_{1}, \ldots, \mathbf{q}_{k}\right)$, of which the corresponding singular values are greater than some given tolerance $t o l_{p o d}$, i.e., $\sigma_{k} / \sigma_{1}>t_{\text {pod }}$ but $\sigma_{k+1} / \sigma_{1} \leq t o l_{\text {pod }}$. To simplify the later presentation, we denote this POD procedure for generating the reduced basis $Q_{t}$ through a given snapshot set $S_{\Xi}^{t}$ by $Q_{t}:=\operatorname{POD}\left(S_{\Xi}^{t}\right)$.

The second kind consists of snapshot selection methods- that is to select $N_{r}$ snapshots $\left\{u_{h}\left(x, c, \xi_{t}^{(j)}\right)\right\}_{j=1}^{N_{r}}$ to construct the reduced basis $Q_{t}$, i.e., $Q_{t}:=\left\{u_{h}\left(x, c, \xi_{t}^{(j)}\right)\right\}_{j=1}^{N_{r}}$ (here the Gram-Schmidt process is typically required to modify the snapshots to retain numerical stability). A variety of methods for selecting snapshots have been developed during the last decade, e.g., greedy sampling methods [46, 47, 48, 49, 38, 50, 51], and optimization based greedy approaches [52]. In this paper, we focus on greedy sampling approaches. The main idea of them is to adaptively select parameter samples from a given training set, where reduced basis approximations have large errors. This typically requires looping over the training set as follows. Taking an input parameter sample, we apply a given current reduced basis to compute a reduced basis approximation solution, and to estimate the error of the reduced basis approximation using some error indicator (or estimator). If the estimated error is larger than a given tolerance, 
the snapshot associated with this input sample is selected to update the current reduced basis. The above procedure is repeated until $N_{r}$ snapshots are obtained.

Error indicators play an important role in the snapshot selection methods. Effective error estimators have been developed in [46, 53, 49]. For simplicity, we in this paper use an algebraic residual error indicator to select snapshots. Following our notation in [6], when considering linear PDEs, the algebraic system associated with 17] can be written as $\mathbf{A}_{\xi_{t}} \mathbf{u}_{\xi_{t}}=\mathbf{f}$ where $\mathbf{A}_{\xi_{t}} \in \mathbb{R}^{N_{h} \times N_{h}}$, and $\mathbf{u}_{\xi_{t}}, \mathbf{f} \in \mathbb{R}^{N_{h}}$. The algebraic system of the reduced basis approximation (18) can be written as $\mathbf{Q}_{t}^{T} \mathbf{A}_{\xi_{t}} \mathbf{Q}_{t} \tilde{\mathbf{u}}_{\xi_{t}}=\mathbf{Q}_{t}^{T} \mathbf{f}$, where $\tilde{\mathbf{u}}_{\xi_{t}} \in$ $\mathbb{R}^{N_{r}}$ gives a reduced basis solution and $\mathbf{Q} \in \mathbb{R}^{N_{h} \times N_{r}}$ is the matrix form of the reduced basis $Q=\left\{q_{1}, \ldots, q_{N_{r}}\right\}$, i.e., each column of $\mathbf{Q}_{t}$ is the vector of nodal coefficient values associated with each $q_{i}, i=1, \ldots, N_{r}$. The residual indicator is defined by

$$
\tau_{\xi_{t}}:=\frac{\left\|\mathbf{A}_{\xi_{t}} \mathbf{Q}_{t} \tilde{\mathbf{u}}_{\xi_{t}}-\mathbf{f}\right\|_{2}}{\|\mathbf{f}\|_{2}}
$$

Cf. [6] for implementation details of the residual error indicator, and [47, 54, 55, 26, 42] for further discussions on reduced basis methods for nonlinear PDEs. In the next section, we present a systematical reduced basis version of Algorithm 1

\section{Reduced basis ANOVA}

We introduce a reduced basis ANOVA method to compute the collocation coefficients $\left\{u\left(x, c, \xi_{t}^{(j)}\right), \xi_{t}^{(j)} \in \Theta_{t}\right\}$ in (12) for each ANOVA index $t \in \mathcal{J}$. Following the reduced basis collocation approach introduced in [6], we use the reduced solution $u_{r}\left(x, c, \xi_{t}^{(j)}\right)$ (see (18)) to serve as the collocation coefficient $u\left(x, c, \xi_{t}^{(j)}\right)$ in (12) whenever the reduced solution is accurate enough. That is, for a given collocation point $\xi_{t}^{(j)} \in \Theta_{t}$, we compute a reduced solution $u_{r}\left(x, c, \xi_{t}^{(j)}\right)$ and the residual indicator $\tau_{\xi_{t}^{(j)}}$ (see (20)). If the residual indicator is smaller than a given tolerance, the reduced solution is used to serve as the collocation coefficient; otherwise, we compute the snapshot $u_{h}\left(x, c, \xi_{t}^{(j)}\right)$ (i.e., the finite element solution in (17)), and use the snapshot to serve as the collocation coefficient, meanwhile we augment the reduced basis with this snapshot. The details of our reduced basis ANOVA approach are described as follows.

First, we set $\mathcal{J}_{0}:=\{\emptyset\}$ and consider the zeroth ANOVA order term $u_{\emptyset}^{\mathrm{sc}}\left(x, \xi_{\emptyset}\right)$ in 
through solving (17) with $\xi_{t}=c$. We construct the zeroth order reduced basis using this snapshot $Q_{\emptyset}:=\left\{u_{h}(x, c)\right\}$, and define a first order index set by $\mathcal{J}_{1}:=\{1, \ldots, M\}$.

Second, we consider an ANOVA order $i \geq 1$. Supposing the index set $\mathcal{J}_{i}$ and the reduced bases for the previous order $\left(Q_{s}\right.$ for all $\left.s \in \mathcal{J}_{i-1}\right)$ are given, the following greedy approach is applied to compute the collocation coefficients of the ANOVA term associated with each $t \in \mathcal{J}_{i}$. To start a greedy procedure, an initial basis is required. To initialize the reduced basis $Q_{t}$ for $t \in \mathcal{J}_{i}$, we introduce a hierarchical approach based on the nested structure of ANOVA indices (see (11)), such that bases generated at the previous order (order $i-1$ ) can be properly reused:

1. grouping all reduced basis functions associated with subindices of $t$ with order $|t|-1$ together, we define $Q_{t}^{0}:=\cup_{s \in \Lambda_{t}} Q_{s}$ where $\Lambda_{t}:=\left\{s \mid s \in \mathcal{J}_{|t|-1}\right.$ and $\left.s \subset t\right\}$;

2. since $Q_{t}^{0}$ may contain linearly dependent terms, we apply POD to $Q_{t}^{0}$ to result in an orthogonal basis, and use this POD basis to serve as an initialization of $Q_{t}$, i.e., we initially set $Q_{t}:=\operatorname{POD}\left(Q_{t}^{0}\right)$ (details of POD are discussed in Section 3 ).

After the initial basis is generated, a collocation sample set $\Theta_{t} \in I^{|t|}$ needs to be specified, e.g., the tensor Clenshaw-Curtis grids [14] and sparse grids [56, 3]. Then, looping over the collocation points, we compute the reduced solution $u_{r}\left(x, c, \xi_{t}^{(j)}\right)$ (see (18) for each $\xi_{t}^{(j)} \in \Theta_{t}$, and the residual indicator through $\tau_{\xi_{t}^{(j)}}$ (see (20)):

1. if the residual indicator is smaller than a given tolerance $t o l_{r b}$, use $u_{r}\left(x, c, \xi_{t}^{(j)}\right)$ to serve as the collocation coefficients in 12 ;

2. if the residual indicator is larger than or equal to $t o l_{r b}$, compute the snapshot $u_{h}\left(x, c, \xi^{(j)}\right)$ through solving [17), use the snapshot to serve as the collocation coefficient and update the reduced basis $Q_{t}$ with this snapshot.

When the collocation coefficients in (12) are generated using the above greedy ap215 proach, the ANOVA expansion term $u_{t}^{\mathrm{sc}}\left(x, \xi_{t}\right)$ can be assembled by (12) and (14). At the end of this step, we compute the relative mean value $\tilde{\gamma}_{t}$ using (16).

Next, the index set for the next ANOVA order $\left(\mathcal{J}_{i+1}\right)$ can be constructed following the method presented in [12]. That is first to remove the indices associated with these small relative mean values, i.e., we define a set $\tilde{\mathcal{J}}_{i}:=\left\{t \mid t \in \mathcal{J}_{i}\right.$, and $\left.\tilde{\gamma}_{t} \geq t o l_{\text {anova }}\right\}$ 

$\left\{t \mid t \in \mathfrak{I}_{i+1}\right.$, and any $s \subset t$ with $|s|=i$ satisfies $\left.s \in \tilde{\mathcal{J}}_{i}\right\}$.

Finally, the above procedure is repeated until no higher order ANOVA index can be constructed, i.e. $\mathcal{J}_{i+1}=\emptyset$.

Our reduced basis ANOVA strategy is formally stated in Algorithm 2 In the fol-

\section{Numerical study}

We in this section first consider diffusion problems, and next consider a Stokes problem in Section 5.5 The governing equations of the diffusion problems are

$$
\begin{aligned}
-\nabla \cdot(a(x, \xi) \nabla u(x, \xi)) & =1 & & \text { in } & & D \times \Gamma, \\
u(x, \xi) & =0 & & \text { on } & & \partial D_{D} \times \Gamma, \\
\frac{\partial u(x, \xi)}{\partial n} & =0 & & \text { on } & & \partial D_{N} \times \Gamma,
\end{aligned}
$$

where $\partial u / \partial n$ is the outward normal derivative of $u$ on the boundaries, $\partial D_{D}$ has positive $(d-1)$-dimensional measure, $\partial D_{D} \cap \partial D_{N}=\emptyset$ and $\partial D=\partial D_{D} \cup \partial D_{N}$. Defining

$H^{1}(D):=\left\{u: D \rightarrow \mathbb{R}, \int_{D} u^{2} \mathrm{~d} D<\infty, \int_{D}\left(\partial u / \partial x_{l}\right)^{2} \mathrm{~d} D<\infty, l=1, \ldots, d\right\}$ and $H_{0}^{1}(D):=$ $\left\{v \in H^{1}(D) \mid v=0\right.$ on $\left.\partial D_{D}\right\}$, the weak form of (21)-(23) is to find $u(x, \xi) \in H_{0}^{1}(D)$ such that $(a \nabla u, \nabla v)=(1, v)$ for all $v \in H_{0}^{1}(D)$. We discretize in space using a bilinear finite element approximation [57, 58].

In the following numerical studies, the spatial domain is taken to be $D=(0,1) \times$ 240 $(0,1)$. Mixed boundary conditions are applied - the condition (22) is applied on the left $(x=0)$ and right $(x=1)$ boundaries, and (23) is applied on the top and bottom boundaries. The problem is discretized in space on a uniform $33 \times 33$ grid (the number of the spatial degrees of freedom $N_{h}=1089$ ). 


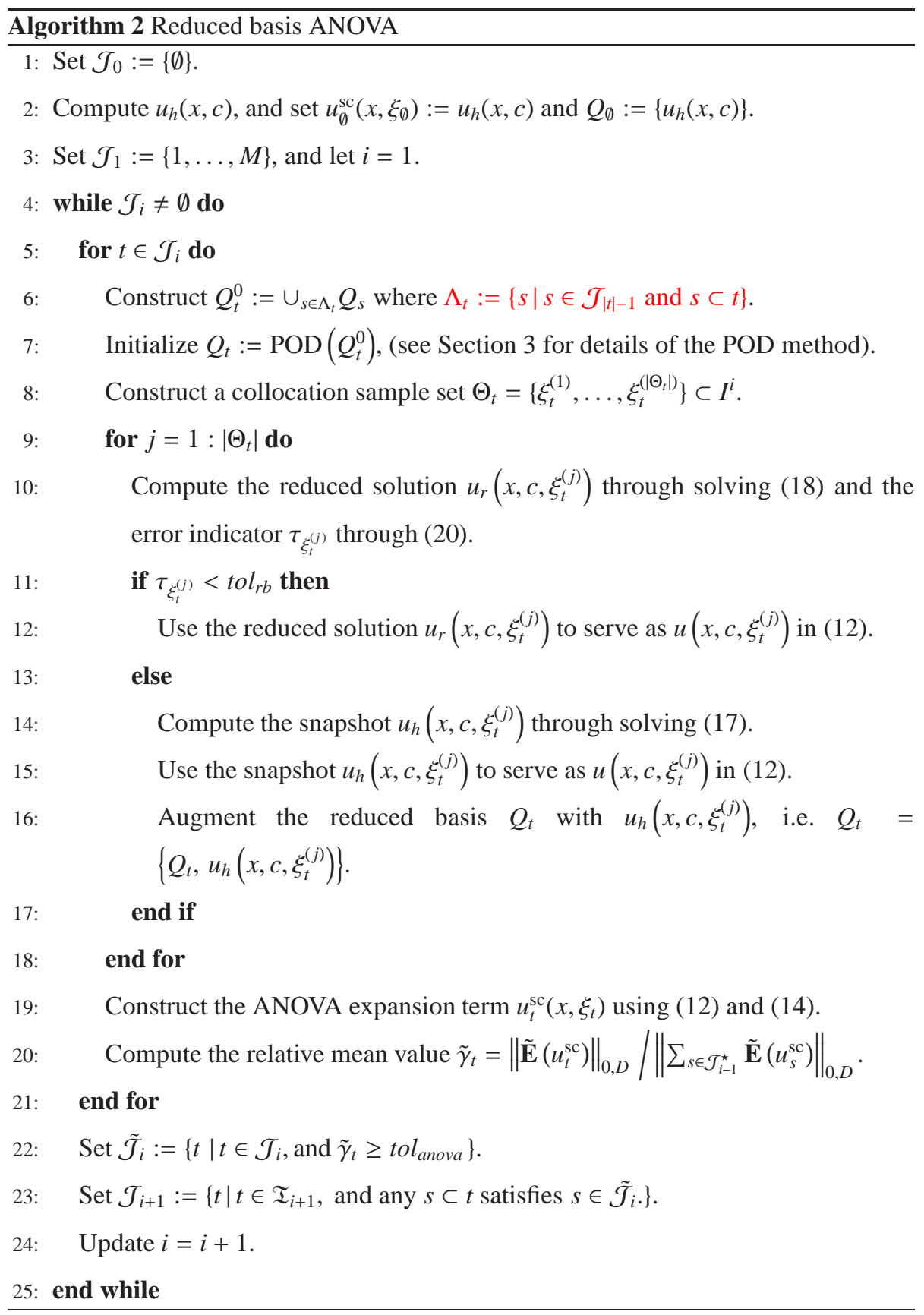


The diffusion coefficient $a(x, \xi)$ in our numerical studies is assumed to be a random

field with mean function $a_{0}(x)$, standard deviation $\sigma$ and covariance function $\operatorname{Cov}(x, y)$,

$$
\operatorname{Cov}(x, y)=\sigma^{2} \exp \left(-\frac{\left|x_{1}-y_{1}\right|}{L}-\frac{\left|x_{2}-y_{2}\right|}{L}\right),
$$

where $x=\left[x_{1}, x_{2}\right]^{T}, y=\left[y_{1}, y_{2}\right]^{T}$ and $L$ is the correlation length. This random field can be approximated by a truncated Karhunen-Loève (KL) expansion [2, 59, 56]

$$
a(x, \xi) \approx a_{0}(x)+\sum_{k=1}^{M} \sqrt{\lambda_{k}} a_{k}(x) \xi_{k},
$$

where $a_{k}(x)$ and $\lambda_{k}$ are the eigenfunctions and eigenvalues of (24), $M$ is the number of KL modes retained, and $\left\{\xi_{k}\right\}_{k=1}^{M}$ are uncorrelated random variables. In this paper, we set the random variables $\left\{\xi_{k}\right\}_{k=1}^{M}$ to be independent uniform distributions with range $I=[-1,1]$.

The error associated with truncation of the KL expansion depends on the amount of total variance captured, $\delta_{K L}:=\left(\sum_{k=1}^{M} \lambda_{k}\right) /\left(|D| \sigma^{2}\right)$, where $|D|$ denotes the area of $D$ [60, 61]. In the following, we set $a_{0}(x)=1$ and $\sigma=0.5$, and examine two test problems with $L=0.625$ and $L=0.3125$ respectively. To satisfy the criterion $\delta_{K L}>95 \%$, we choose $M=73$ for $L=0.625$, and $M=367$ for $L=0.3125$.

\subsection{Moment estimation and comparison}

To assess the accuracy of standard and reduced basis ANOVA-collocation approximations, we compute their mean and variance functions and compare them with reference results as follows.

For the mean function of $u_{\mathcal{J}}^{\text {sc }}(x, \xi)$ (see (13)), we compute it through

$$
\tilde{\mathbf{E}}\left(u_{\mathcal{J}}^{\mathrm{sc}}(x, \xi)\right)=\sum_{t \in \mathcal{J}} \tilde{\mathbf{E}}\left(u_{t}^{\mathrm{sc}}\left(x, \xi_{t}\right)\right)
$$

where the (collocation) quadrature $\tilde{\mathbf{E}}(\cdot)$ is defined in 15 . Note that the equation 26 holds, since we use tensor style quadrature. In the following numerical studies, the tensor style Clenshaw-Curtis quadrature $[62,14]$ with $9^{|t|}$ collocation (quadrature) points are used for each $t \in \mathcal{J}$, i.e. $\left|\Theta_{t}\right|=9^{|t|}$. 
Following the method proposed in [63], we compute the variance function of $u_{\mathcal{J}}^{\mathrm{sc}}(x, \xi)$ as follows

$$
\begin{aligned}
\tilde{\mathbf{V}}\left(u_{\mathcal{J}}^{\mathrm{sc}}(x, \xi)\right) & :=\tilde{\mathbf{E}}\left(\left(u_{\mathcal{J}}^{\mathrm{sc}}(x, \xi)-\tilde{\mathbf{E}}\left(u_{\mathcal{J}}^{\mathrm{sc}}(x, \xi)\right)\right)^{2}\right) \\
& =\tilde{\mathbf{E}}\left(\left(\sum_{t \in \mathcal{T}} u_{t}^{\mathrm{sc}}\left(x, \xi_{t}\right)-\sum_{t \in \mathcal{T}} \tilde{\mathbf{E}}\left(u_{t}^{\mathrm{sc}}\left(x, \xi_{t}\right)\right)\right)^{2}\right) \\
& =\tilde{\mathbf{E}}\left(\left(\sum_{t \in \mathcal{T}}\left(u_{t}^{\mathrm{sc}}\left(x, \xi_{t}\right)-\tilde{\mathbf{E}}\left(u_{t}^{\mathrm{sc}}\left(x, \xi_{t}\right)\right)\right)\right)^{2}\right) \\
& =\tilde{\mathbf{E}}\left(\left(\sum_{t \in \mathcal{T}}\left(u_{t}^{\mathrm{sc}}\left(x, \xi_{t}\right)-\tilde{\mathbf{E}}\left(u_{t}^{\mathrm{sc}}\left(x, \xi_{t}\right)\right)\right)\right)\left(\sum_{s \in \mathcal{T}}\left(u_{s}^{\mathrm{sc}}\left(x, \xi_{s}\right)-\tilde{\mathbf{E}}\left(u_{s}^{\mathrm{sc}}\left(x, \xi_{s}\right)\right)\right)\right)\right) \\
& =\sum_{t, s \in \mathcal{T}} \tilde{\mathbf{E}}\left(\left(u_{t}^{\mathrm{sc}}\left(x, \xi_{t}\right)-\tilde{\mathbf{E}}\left(u_{t}^{\mathrm{sc}}\left(x, \xi_{t}\right)\right)\right)\left(u_{s}^{\mathrm{sc}}\left(x, \xi_{s}\right)-\tilde{\mathbf{E}}\left(u_{s}^{\mathrm{sc}}\left(x, \xi_{s}\right)\right)\right)\right) . \quad(27)
\end{aligned}
$$

Note that (27) means adding together covariances of all pairs of the ANOVA expansion terms in (13) [63]. In addition, strategies of computing higher-order statistical moments are also developed in [63].

For comparison, Monte Carlo simulation is considered. Solution samples generated through the Monte Carlo simulation for (11)-(2) with $N$ samples are denoted by $\left\{u^{\mathrm{mc}}\left(x, \xi^{(j)}\right)\right\}_{j=1}^{N}$. The Monte Carlo mean and variance estimates are computed as follows

$$
\begin{aligned}
\mathbf{E}_{N}\left(u^{\mathrm{mc}}\right) & :=\sum_{j=1}^{N} \frac{u^{\mathrm{mc}}\left(x, \xi^{(j)}\right)}{N} \\
\mathbf{V}_{N}\left(u^{\mathrm{mc}}\right) & :=\sum_{j=1}^{N} \frac{1}{N}\left(u^{\mathrm{mc}}\left(x, \xi^{(j)}\right)-\mathbf{E}_{N}\left(u^{\mathrm{mc}}\right)\right)^{2} .
\end{aligned}
$$

In order to generate the solution samples $\left\{u^{\mathrm{mc}}\left(x, \xi^{(j)}\right)\right\}_{j=1}^{N}$, we consider both the finite element method and a direct reduced basis approach to solve the deterministic version of (11-(2). Without confusion, we also use (17) and (18) to denote the finite element and the reduced basis formulations for the global problem (10-(2), while we use $u_{h}\left(x, \xi^{(j)}\right)$ and $u_{r}\left(x, \xi^{(j)}\right.$ ) (for $\left.j=1, \ldots, N\right)$ to denote a snapshot and a reduced basis solution for the global problem respectively. Similarly following the notation in 20 , we use $\tau_{\xi^{(j)}}$ to denote the residual error indicator for the global problem.

In the following, the Monte Carlo solution samples generated by the finite ele- 
ment method are denoted by $\left\{u_{h}^{\mathrm{mc}}\left(x, \xi^{(j)}\right)\right\}_{j=1}^{N}$, and the estimated mean function (28) and the estimated variance function (29) associated with them are denoted $\mathbf{E}_{N}\left(u_{h}^{\mathrm{mc}}\right)$ and $\mathbf{V}_{N}\left(u_{h}^{\mathrm{mc}}\right)$ respectively. We refer to this Monte Carlo method (only using finite element solution samples) as the standard Monte Carlo method. By setting a large sample size $N_{\text {ref }}=10^{8}$, we generate reference mean and variance functions, which are denoted by $\mathbf{E}_{N_{\text {ref }}}\left(u_{h}^{\mathrm{mc}}\right)$ and $\mathbf{V}_{N_{\text {ref }}}\left(u_{h}^{\mathrm{mc}}\right)$ respectively.

Applying reduced basis methods to generate solution samples in Monte Carlo simulation is studied in detail in [49]. For simplicity, we in this paper only consider a direct combination of reduced basis methods and Monte Carlo methods for comparison. This (direct) reduced basis Monte Carlo approach is stated in Algorithm 3 We denote the Monte Carlo solution samples generated by Algorithm 3 by $\left\{u_{r}^{\mathrm{mc}}\left(x, \xi^{(j)}\right\}_{j=1}^{N}\right.$. The mean function and the variance function computed through putting $\left\{u_{r}^{\mathrm{mc}}\left(x, \xi^{(j)}\right)\right\}_{j=1}^{N}$ into (28)-290 are denoted $\mathbf{E}_{N}\left(u_{r}^{\mathrm{mc}}\right)$ and $\mathbf{V}_{N}\left(u_{r}^{\mathrm{mc}}\right)$ respectively.

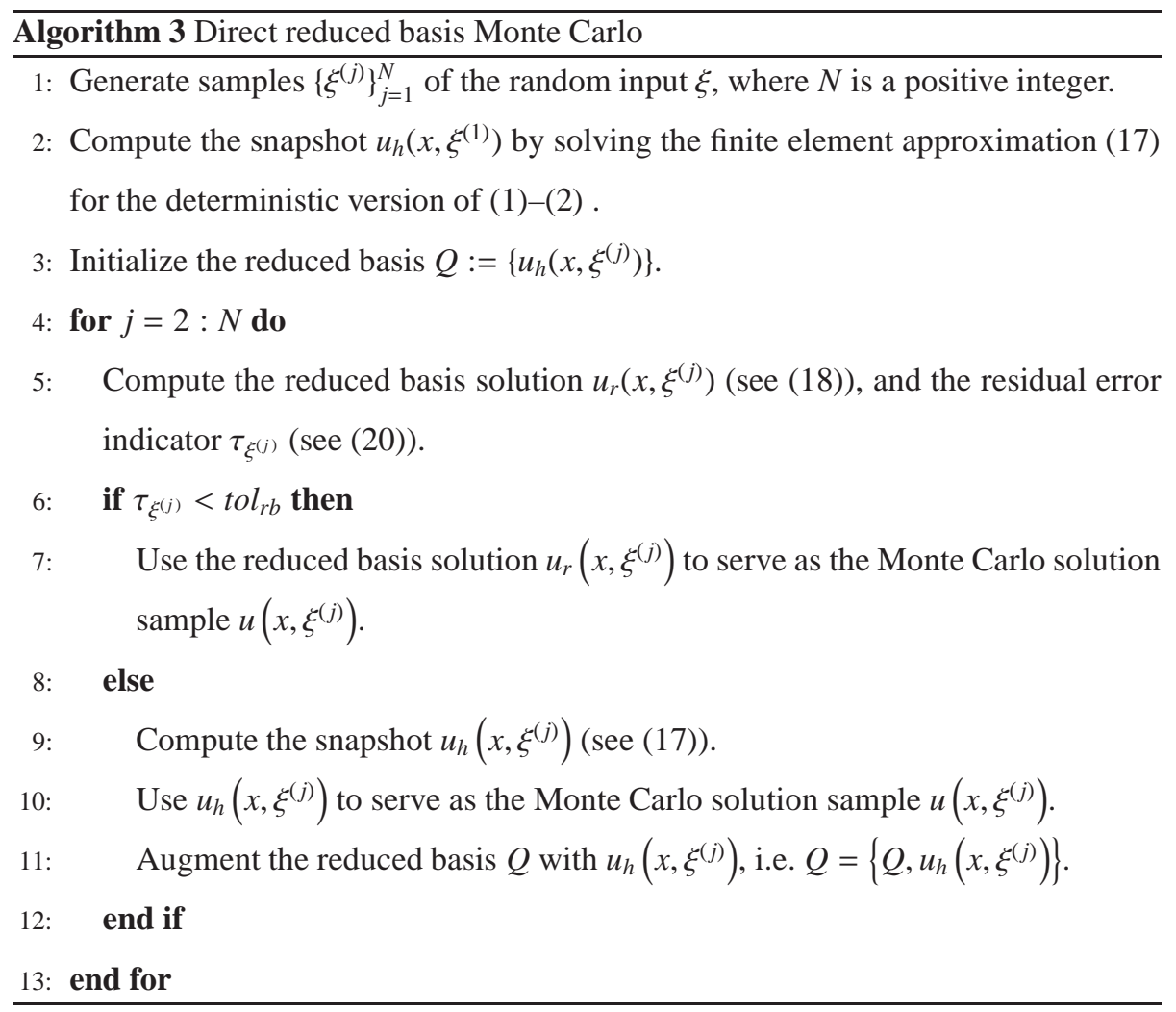


The errors of mean and variance functions estimated through the above ANOVA and Monte Carlo methods are evaluated as follows. For the standard and the reduced basis ANOVA methods, the following quantities are introduced,

$$
\begin{aligned}
\epsilon_{h} & :=\left\|\tilde{\mathbf{E}}\left(u_{\mathcal{J}}^{h \mathrm{sc}}\right)-\mathbf{E}_{N_{\text {ref }}}\left(u_{h}^{\mathrm{mc}}\right)\right\|_{0} /\left\|\mathbf{E}_{N_{\text {ref }}}\left(u_{h}^{\mathrm{mc}}\right)\right\|_{0}, \\
\epsilon_{r} & :=\left\|\tilde{\mathbf{E}}\left(u_{\mathcal{J}}^{r \mathrm{sc}}\right)-\mathbf{E}_{N_{\text {ref }}}\left(u_{h}^{\mathrm{mc}}\right)\right\|_{0} /\left\|\mathbf{E}_{N_{\text {ref }}}\left(u_{h}^{\mathrm{mc}}\right)\right\|_{0}, \\
\eta_{h} & :=\left\|\tilde{\mathbf{V}}\left(u_{\mathcal{J}}^{h \mathrm{sc}}\right)-\mathbf{V}_{N_{\text {ref }}}\left(u_{h}^{\mathrm{mc}}\right)\right\|_{0} /\left\|\mathbf{V}_{N_{\text {ref }}}\left(u_{h}^{\mathrm{mc}}\right)\right\|_{0}, \\
\eta_{r} & :=\left\|\tilde{\mathbf{V}}\left(u_{\mathcal{J}}^{r \mathrm{sc}}\right)-\mathbf{V}_{N_{\text {ref }}}\left(u_{h}^{\mathrm{mc}}\right)\right\|_{0} /\left\|\mathbf{V}_{N_{\text {ref }}}\left(u_{h}^{\mathrm{mc}}\right)\right\|_{0} .
\end{aligned}
$$

Similarly, for $N<N_{\text {ref }}$, errors of Monte Carlo methods are assessed by

$$
\begin{aligned}
& \tilde{\epsilon}_{h}:=\left\|\mathbf{E}_{N}\left(u_{h}^{\mathrm{mc}}\right)-\mathbf{E}_{N_{\text {ref }}}\left(u_{h}^{\mathrm{mc}}\right)\right\|_{0} /\left\|\mathbf{E}_{N_{\text {ref }}}\left(u_{h}^{\mathrm{mc}}\right)\right\|_{0}, \\
& \tilde{\epsilon}_{r}:=\left\|\mathbf{E}_{N}\left(u_{r}^{\mathrm{mc}}\right)-\mathbf{E}_{N_{\mathrm{ref}}}\left(u_{h}^{\mathrm{mc}}\right)\right\|_{0} /\left\|\mathbf{E}_{N_{\mathrm{ref}}}\left(u_{h}^{\mathrm{mc}}\right)\right\|_{0}, \\
& \tilde{\eta}_{h}:=\left\|\mathbf{V}_{N}\left(u_{h}^{\mathrm{mc}}\right)-\mathbf{V}_{N_{\mathrm{ref}}}\left(u_{h}^{\mathrm{mc}}\right)\right\|_{0} /\left\|\mathbf{V}_{N_{\text {ref }}}\left(u_{h}^{\mathrm{mc}}\right)\right\|_{0}, \\
& \tilde{\eta}_{r}:=\left\|\mathbf{V}_{N}\left(u_{r}^{\mathrm{mc}}\right)-\mathbf{V}_{N_{\text {ref }}}\left(u_{h}^{\mathrm{mc}}\right)\right\|_{0} /\left\|\mathbf{V}_{N_{\text {ref }}}\left(u_{h}^{\mathrm{mc}}\right)\right\|_{0} .
\end{aligned}
$$

For a fair comparison between the standard and the reduced basis Monte Carlo methods with a given sample size $N$, we use the same input sample set $\left\{\xi^{(j)}\right\}_{j=1}^{N}$ for them.

In Algorithm 2, there exist three tolerance parameters that need to be specified. The first one does not explicitly appear but it exists in line 7 of Algorithm 2 which is $t_{\text {ol }}$ (see Section 3 ) to select singular vectors in POD. We set $\operatorname{tol}_{p o d}=10^{-3}$ for our test problems (we also tested smaller values of $t_{\text {pod }}$, and no significantly different results were found). The second is $t o l_{r b}$ for selecting snapshots (line 11 of Algorithm 2) - we test three cases for this tolerance parameter: $t_{0 l} r=10^{-4}, 10^{-5}$, and $10^{-6}$ in the following numerical studies. The last one is $t_{\text {anova }}$ for selecting active ANOVA indices (line 22 of Algorithm 2). The effect of choosing different values of tol anova is studied in detail in $\left\lfloor 12,\lfloor 14\rfloor\right.$, and we set tol $l_{\text {anova }}=10^{-4}$ here.

As discussed above, we take fixed tolerance parameter values except for the residual error tolerance $t o l_{r b}$ to test Algorithm 2, To indicate the different choices of $t o l_{r b}$, we refine our notation of the error quantities (31) and (33) by adding the $t o l_{r b}$ to them$\epsilon_{r}\left(t_{o l}\right)$ denotes the mean function error of the reduced basis ANOVA associated with 

basis ANOVA associated with the tolerance $t o l_{r b}$. In addition, since the residual error tolerance $t l_{r b}$ also exists in the reduced basis Monte Carlo method (line 6 of Algorithm 3), the error quantity notation in (35) and (37) are also refined- $\tilde{\epsilon}_{r}\left(\operatorname{tol}_{r b}\right)$ and $\tilde{\eta}_{r}\left(\operatorname{tol}_{r b}\right)$ are used to denote relative mean and variance function errors of the reduced basis

\subsection{Computational cost assessment}

The main cost of generating the ANOVA-collocation approximation (13) comes from computing the collocation coefficients in (12), which are solutions of the deterministic version of (7)-(8) at collocation points. To assess the costs, we first count relative sizes of linear systems and develop a simple computational cost model, so as to provide a cost measure independent of computational platforms. CPU times of the corresponding linear system solves are also presented in the following for comparison.

For a given number of finite element degrees of freedom $N_{h}$, we assume that the costs for computing all snapshots (i.e., solving (17) with respect to different realizations of the random inputs) are equal for simplicity. We define a cost unit by the cost for computing each snapshot. The cost for generating the standard ANOVA-collocation approximation can then be written as $\sum_{t \in \mathcal{J}}\left|\theta_{t}\right|$, while the cost of the standard Monte Carlo method with $N$ samples is $N$ with respect to the cost unit.

As in standard complexity analysis, costs of using direct methods to solve alge335 braic versions of 17 and 18 are $O\left(N_{h}^{3}\right)$ and $O\left(N_{r}^{3}\right)$ respectively, while the costs can be reduced to $O\left(N_{h}\right)$ and $O\left(N_{r}^{2}\right)$ through using optimal iterative methods (see [41]). Since the performances of iterative methods are dependent on preconditioners [58, 41], it remains an open question to accurately measure optimal costs of solving reduced problems relative to the cost of computing each snapshot (the cost unit). By counting 340 by $N_{r} / N_{h}$ for simplicity. When considering the reduced basis Monte Carlo method (Algorithm 3), since the reduced basis size $N_{r}$ can vary between different input samples during the greedy procedure, we here use $N_{r}\left(\xi^{(j)}\right), j=1, \ldots, N$, to denote the size of the reduced basis associated with each input sample $\xi^{(j)}$. The cost of the reduced 
written as $\tilde{N}+\sum_{j=1}^{N} N_{r}\left(\xi^{(j)}\right) / N_{h}$. In the same way, we model the cost of the reduced basis ANOVA approach (Algorithm 2) - the cost is set to be the sum of the costs of full system solves and the costs of reduced system solves assessed above. Cf. [64, 65] for detailed discussions about measuring computational costs associated with mixed (or multifidelity) methods.

In addition to the cost model introduced above, the CPU times of each method (ANOVA or Monte Carlo) are assessed by adding together the CPU times of all linear system solves involved in each method. In the following numerical studies, all linear systems are solved using the MATLAB "backslash" operator on a MAC Pro with 3.5

\subsection{Results of standard and reduced basis Monte Carlo methods}

To address the challenge in solving the stochastic diffusion problem (21)-(23) with small correlation lengths, we first apply Monte Carlo methods to solve our test problems. Figure 1 shows the mean and the variance function errors of the standard and Section 5.2

From the results of the test problem with $L=0.625$ (Figure 1 (a) and Figure 1(c)), the reduced basis Monte Carlo method associated with residual error tolerances $t l_{r b}=$ $10^{-4}$ and $t o l_{r b}=10^{-5}$ is slightly cheaper than the standard Monte Carlo method to achieve the same mean and variance error values. However, when choosing $t^{\circ} l_{r b}=$ $10^{-6}$ for this test problem, the cost of the reduced basis Monte Carlo is very close to that of the standard Monte Carlo. For the results of the test problem with $L=0.3125$ (Figure 1(b) and Figure 1(d)), costs of the reduced basis Monte Carlo with different choices of the residual error tolerance are close to the cost of the standard Monte Carlo method.

As studied in [6], performance of reduced basis methods is dependent on the rank of the full snapshot set $S_{I^{M}}:=\left\{u_{h}(x, \xi), \xi \in I^{M}\right\}$. When the rank of $S_{I^{M}}$ is much smaller than the finite element degrees of freedom $N_{h}$, the reduced basis method is efficient; otherwise, it may not be efficient. To understand the difficulty of the two 

$\Xi$ consisting of $10^{4}$ samples uniformly distributed in $I^{M}$, and then construct a finite snapshot set $S_{\Xi}:=\left\{u_{h}(x, \xi), \xi \in \Xi\right\}$. After that, the matrix form of $S_{\Xi}$ is denoted by $\mathbf{S}_{\Xi} \in \mathbb{R}^{N_{h} \times|\Xi|}$, where each column of $\mathbf{S}_{\Xi}$ is the vector of basis function coefficients of a finite element solution. Finally, perform SVD of $\mathbf{S}_{\Xi}$ and count the number of singular

values larger than a given tolerance $t o l_{\text {rank }}$. That is, let $\mathbf{S}_{\Xi}=U \Sigma V^{T}$ be the SVD of $\mathbf{S}_{\Xi}$, where $\Sigma=\operatorname{diag}\left(\sigma_{1}, \cdots, \sigma_{N_{h}}\right)$ with $\sigma_{1} \geq \sigma_{2} \geq \cdots \geq \sigma_{N_{h}} \geq 0$ (we take $|\Xi|>N_{h}$ to access the ranks), and the estimated rank is defined by $k$ such that $\sigma_{k} / \sigma_{1}>\operatorname{tol}_{\text {rank }}$ but $\sigma_{k+1} / \sigma_{1} \leq$ tol $_{\text {rank }}$.

The estimated ranks for the two test problems are presented in Table 1 Compared with the spatial degrees of freedom $\left(N_{h}=1089\right)$, these estimated ranks are not small. Especially, when we set $t o l \leq 10^{-8}$, the diffusion problem with $L=0.3125$ is nearly full of rank, i.e., the estimated rank of its full snapshot set is close to $N_{h}$. In the following, we call the problems, of which the full snapshot sets have ranks close to $N_{h}$, the large-rank problems. As discussed in our earlier work [6] and Section 1 of this paper, large-rank problems are challenging for applying reduced basis methods, which is consistent with our results in Figure 1. To explore low-rank structures in these large-rank problems, we apply the ANOVA approach [12, 14] to decompose the global system (11-(2) into a series of local systems (7)-(8), of which full snapshot sets are expected to have small ranks and detailed numerical studies are in the next section.

Table 1: Estimated ranks for the full snapshot set $S_{I^{M}}$ for both test problems, with $N_{h}=1089$.

\begin{tabular}{c|c|c}
\hline tol $_{\text {rank }}$ & 0.625 & 0.3125 \\
\hline $10^{-7}$ & 433 & 944 \\
\hline $10^{-8}$ & 667 & 1023 \\
\hline $10^{-9}$ & 861 & 1023 \\
\hline
\end{tabular}



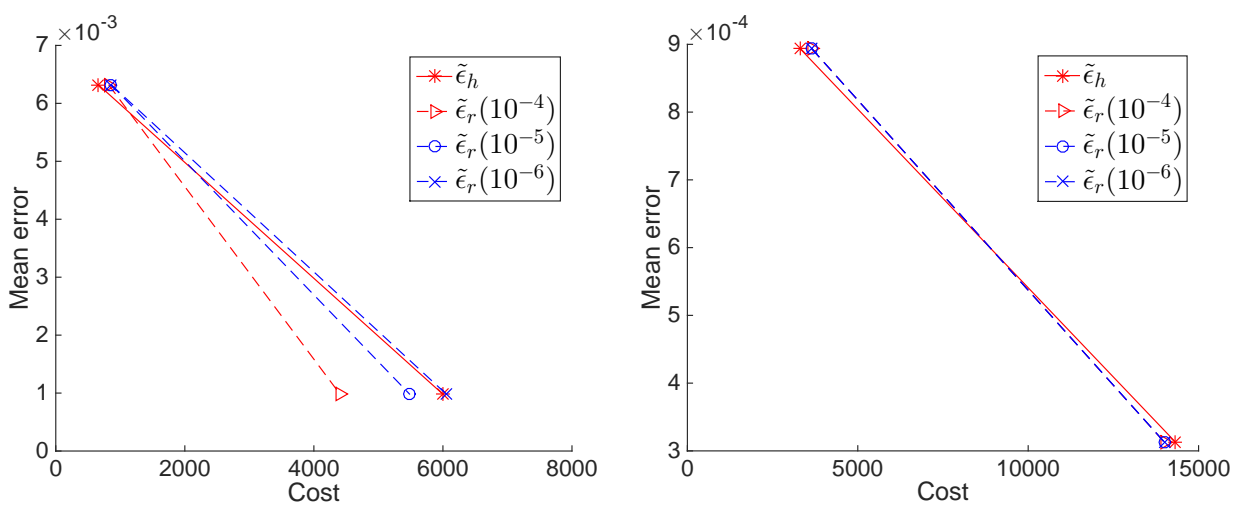

(a) Maan arrorc $I-0675$

(h) Maan arrore $I-\cap 2125$
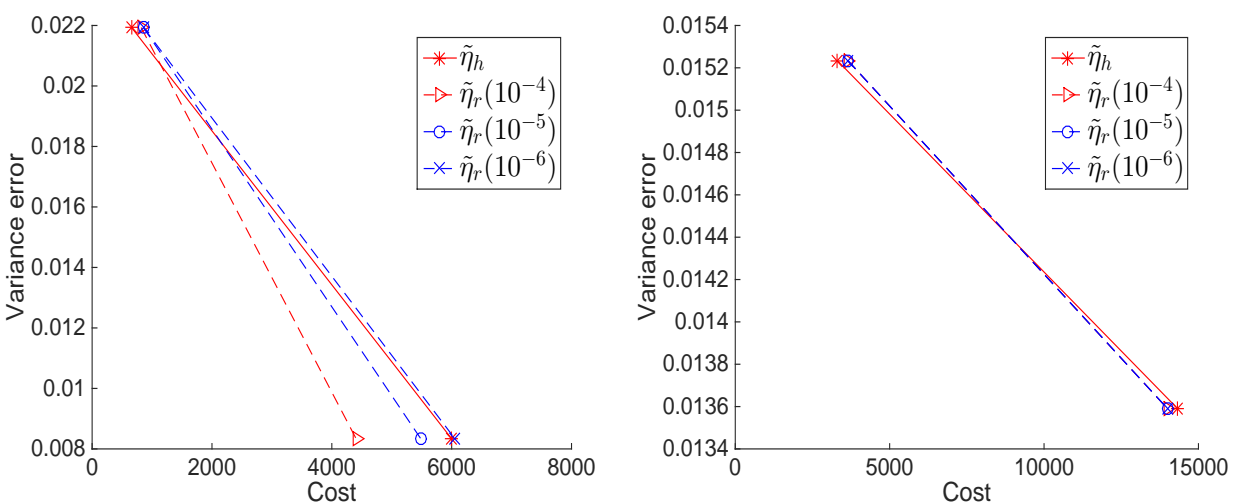

(c) Variance errors, $L=0.625$

(d) Variance errors, $L=0.3125$

Figure 1: Errors in mean and variance function estimates of the standard Monte Carlo ( $\tilde{\epsilon}_{h}$ and $\left.\tilde{\eta}_{h}\right)$ are compared with errors of the reduced basis Monte Carlo $\left(\tilde{\epsilon}_{r}\left(t o l_{r b}\right)\right.$ and $\tilde{\eta}_{r}\left(t o l_{r b}\right)$ associated with different values of the residual error tolerance: $\operatorname{tol}_{r b}=10^{-4}, 10^{-5}, 10^{-6}$ ). 

2], we assess the ranks of the full snapshot sets associated with local problems (7)- (8) for all $t \in \mathcal{J}$, and we refer to these ranks as the local ranks in the following. We assess the local ranks using the same way that we assess the ranks for the global problem discussed in Section 5.3, where SVD is performed on a snapshot set consisting of 425

\subsection{Results of standard and reduced basis ANOVA methods}

In this section, we first apply the standard ANOVA method (Algorithm 1) to solve the two test problems, and then explore low-rank structures in the collocation coefficients in each of the ANOVA terms (12). The numerical efficiency of our reduced basis ANOVA approach is reported finally.

We set the relative mean value tolerance tol $_{\text {anova }}=10^{-4}$ for Algorithm 1 (see [12, 14] for detailed studies on different choices of $t o l_{\text {anova }}$ ). Table 2 shows sizes of the index sets $\left\{\mathcal{J}_{i}\right\}_{i=1,2}$ for constructing the overall ANOVA-collocation approximation (13), and sizes of the selected index sets $\left\{\tilde{\mathcal{J}}_{i}\right\}_{i=1,2}$ (see line 13 of Algorithm 1). It can be seen that only a small percentage of the first order ANOVA indices are selected to construct the second order indices. Moreover, there is no second order index selected for constructing a third order one associated with tol anova $=10^{-4}$, which is consistent with the results in [14]. Since $\tilde{\mathcal{J}}_{2}=\emptyset$ in Table 2 leads to $\mathcal{J}_{i}=\emptyset$ for $i=3, \ldots, M$ (see (11)), we only exam the errors of the standard ANOVA-collocation approximation associated with ANOVA orders $i=1,2$, i.e., errors of $u_{\mathcal{J}}^{h s c}$ with $\mathcal{J}=\mathcal{J}_{1}^{\star}$ and $\mathcal{J}=\mathcal{J}_{2}^{\star}$ respectively (see Section 2.1 for notation).

Next, Figure 2 shows the mean and the variance function errors of the standard ANOVA-collocation approximation $u_{\mathcal{J}}^{h s c}$ for the two test problems. From Figure 2 (a) and Figure 2 b), the standard ANOVA has smaller mean function errors compared with the standard and the reduced basis Monte Carlo methods. However, from Figure 2(c) and Figure 2(d), when considering the first order ANOVA approximation $(i=1)$, errors in the variance function estimates of the standard ANOVA are larger than the errors of the Monte Carlo methods with similar costs. When considering the second order ANOVA approximation $(i=2)$, the standard ANOVA and the Monte Carlo methods with similar costs have very close errors.

Before presenting the results of our reduced basis ANOVA approach (Algorithm $10^{4}$ samples and tol $_{\text {rank }}$ denotes the tolerance to identify the ranks. To plot the local 

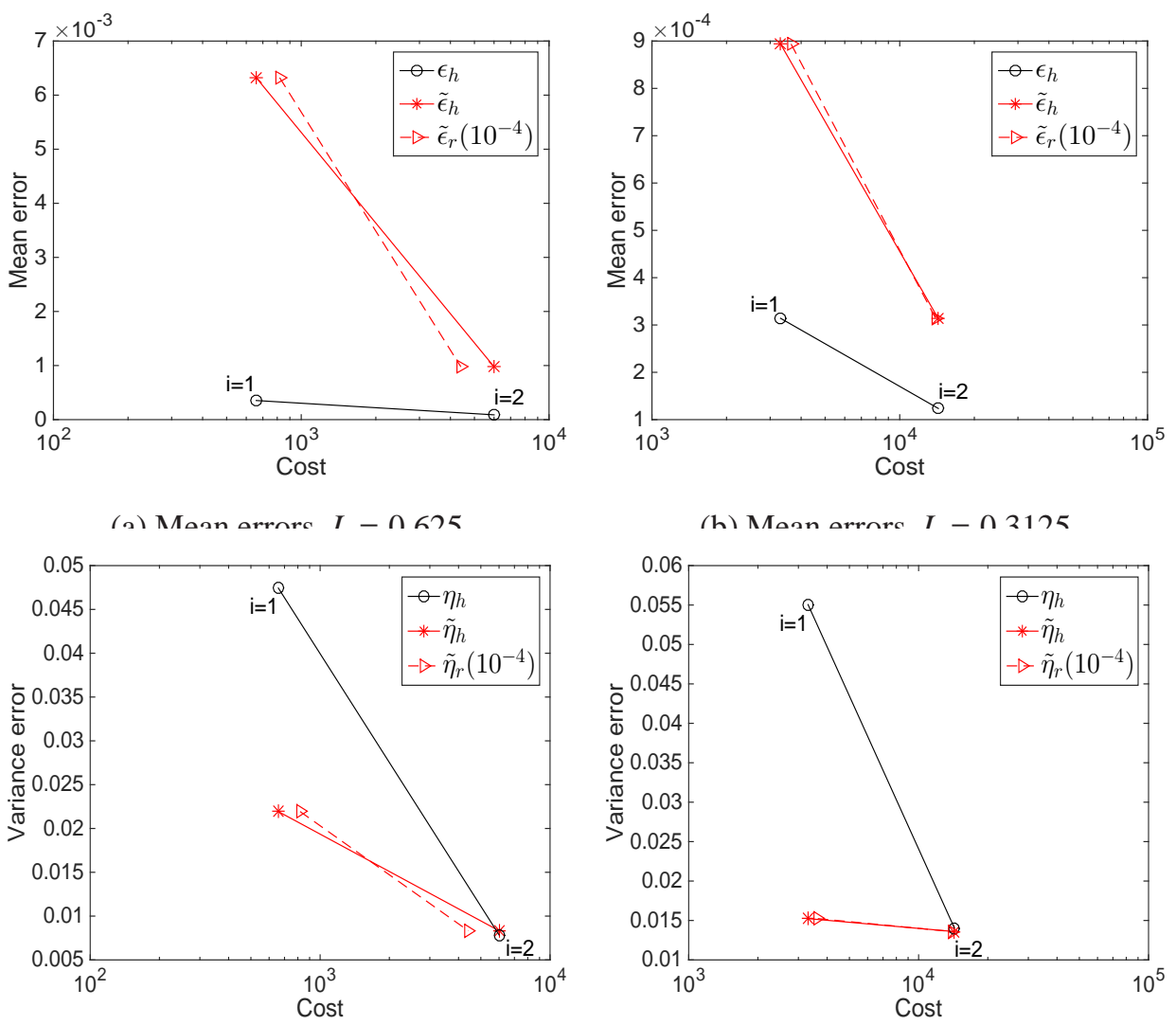

(c) Variance errors, $L=0.625$

(d) Variance errors, $L=0.3125$

Figure 2: Errors in mean and variance function estimates of the standard ANOVA $\left(\epsilon_{h}\right.$ and $\left.\eta_{h}\right)$, are compared with errors of the standard Monte Carlo $\left(\tilde{\epsilon}_{h}\right.$ and $\left.\tilde{\eta}_{h}\right)$ and errors of the reduced basis Monte Carlo $\left(\tilde{\epsilon}_{r}\left(10^{-4}\right)\right.$ and $\left.\tilde{\eta}_{r}\left(10^{-4}\right)\right)$.

Table 2: Size of the ANOVA index set $\left|\mathcal{J}_{i}\right|$ and size of the selected index set $\left|\tilde{\mathcal{T}}_{i}\right|$ at each ANVOA order $i=1,2$.

\begin{tabular}{c|cccc}
\hline$L$ & $\left|\mathcal{J}_{1}\right|$ & $\left|\tilde{\mathcal{J}}_{1}\right|$ & $\left|\mathcal{J}_{2}\right|$ & $\left|\tilde{\mathcal{J}}_{2}\right|$ \\
\hline 0.625 & 73 & 12 & 66 & 0 \\
\hline 0.3125 & 367 & 17 & 136 & 0 \\
\hline
\end{tabular}


ranks associated with each $t \in \mathcal{J}$, we label the indices as $\mathcal{J}=\left\{t^{(1)}, \ldots, t^{(\mathcal{I J})}\right\}$, where the indices are sorted in alphabetical order as follows: considering any two different indices $t^{(j)}$ and $t^{(k)}$ belonging $\mathcal{J}, t^{(j)}$ is ordered before $t^{(k)}$ (i.e., $j<k$ ), if one of the following two cases is true: (a) $\left|t^{(j)}\right|<\left|t^{(k)}\right|$; (b) $\left|t^{(j)}\right|=\left|t^{(k)}\right|$ and for the smallest number ${ }_{430} m \in\left\{1, \ldots,\left|t^{(j)}\right|\right\}$ such that $t_{m}^{(j)} \neq t_{m}^{(k)}$, we have $t_{m}^{(j)}<t_{m}^{(k)}$ (where $t_{m}^{(j)}$ and $t_{m}^{(k)}$ are the $m$-th components of $t^{(j)}$ and $t^{(k)}$ defined in Section 2.11).

Figure 3 shows the estimated local ranks with respect to the index labels defined above. For both test problems, the estimated local ranks are much smaller than $N_{h}=$ 1089, while the estimated ranks associated with the first ANOVA terms are smaller than those associated with the second order terms, which is consistent with the results in [6] - higher parameter space dimensions lead to larger spatial approximation ranks. The maximum and the average of the estimated local ranks are shown in Table 3 , where $R_{t}$ denotes the local rank associated with the index $t \in \mathcal{J}$, and $\bar{R}_{\mathcal{J}_{i}}:=\left(\sum_{t \in \mathcal{J}_{i}} R_{t}\right) /\left|\mathcal{J}_{i}\right|$, $i=1,2$, denotes the average rank over $\mathcal{J}_{i}$ (the average ranks are rounded to the nearest integer). From Table 3 it can be seen that the average and the maximum local ranks for $L=0.625$ are similar to those for $L=0.3125$. As discussed in $\llbracket 6 \rrbracket$, the local ranks mainly depend on local input dimensions (i.e. $|t|$ in (7)-(8). Since $|t|$ equals to the ANOVA order and is independent of the correlation length $L$, we have similar local ranks for both $L=0.625$ and $L=0.3125$ in Table 3 . The ranks of the global 445 problem in Table 1 however, is dependent on the correlation lengths, since different correlation lengths give different input dimensions for the global problem $(M=73$ for $L=0.625$ and $M=367$ for $L=0.3125$ ). Looking at Table 3 in more detail, we see that the maximum local ranks for the first ANOVA order for both test problems are not larger than 6 (with $t o l_{r a n k}=10^{-9}$ ), and those for the second order are less than 25. Unsurprisingly, the averages of the local ranks are smaller than the maxima-they are around 4 for the first ANOVA order, and 16 for the second ANOVA order. This indicates that, in average, no more than 4 snapshots are required to generate accurate reduced basis approximations for collocation coefficients of the first order ANOVA terms, and no more than 16 snapshots are required for the second order terms. Given our collocation sample sizes $\left(\left|\Theta_{t}\right|=9^{|t|}, t \in \mathcal{J}\right)$ which are larger than the average ranks (especially when $|t|=2$ ), we can deduce that applying reduced basis methods can 


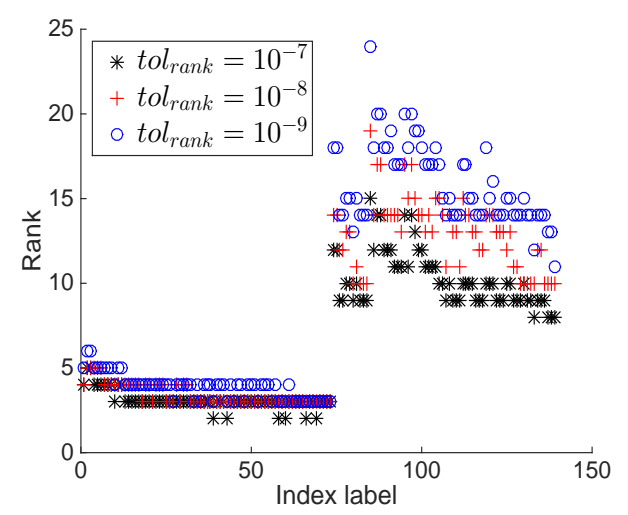

(a) $L=0.625$

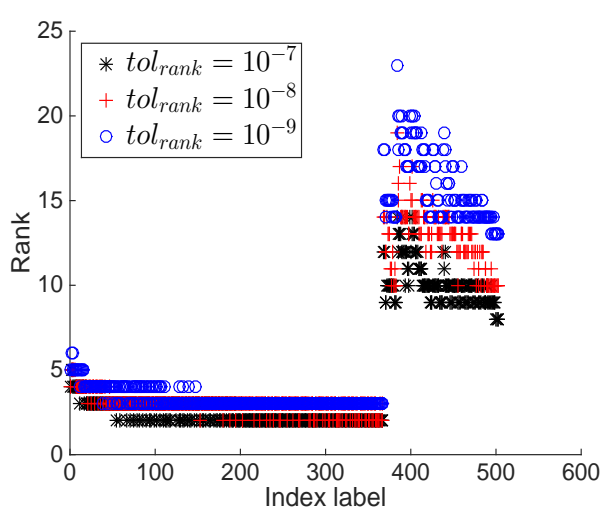

(b) $L=0.3125$

Figure 3: Estimated ranks for both test problems associated with different tolerance values tol $_{\text {rank }}=$ $10^{-7}, 10^{-8}$ and $10^{-7}$.

reduce the costs of the standard ANOVA method, and we show the results next.

Table 3: Maxima and averages of the estimated ranks $\left(\max _{t \in \mathcal{J}_{i}} R_{t}\right.$ and $\left.\bar{R}_{\mathcal{J}_{i}}\right)$ associated with each ANOVA order $i=1,2$.

\begin{tabular}{c|cccc|cccc}
\hline & \multicolumn{4}{|c|}{$L=0.625$} & \multicolumn{5}{c}{$L=0.3125$} \\
tol $_{\text {rank }}$ & $\max _{t \in \mathcal{J}_{1}} R_{t}$ & $\max _{t \in \mathcal{J}_{2}} R_{t}$ & $\bar{R}_{\mathcal{J}_{1}}$ & $\bar{R}_{\mathcal{J}_{2}}$ & $\max _{t \in \mathcal{J}_{1}} R_{t}$ & $\max _{t \in \mathcal{J}_{2}} R_{t}$ & $\bar{R}_{\mathcal{J}_{1}}$ & $\bar{R}_{\mathcal{J}_{2}}$ \\
\hline $10^{-7}$ & 5 & 15 & 3 & 10 & 5 & 14 & 2 & 10 \\
\hline $10^{-8}$ & 5 & 19 & 3 & 13 & 5 & 19 & 3 & 13 \\
\hline $10^{-9}$ & 6 & 24 & 4 & 16 & 6 & 23 & 3 & 16 \\
\hline
\end{tabular}

The mean and variance function errors of the reduced basis ANOVA-collocation approximation associated with different residual error tolerance values are shown in Figure 4. It is clear that, for a given ANOVA order $i=1$ or 2 , the reduced basis ANOVA and the standard ANOVA have visually the same mean and variance errors, while the costs of the reduced basis ANOVA are around only ten percent of the costs of the standard ANOVA. Figure 4 (a) and Figure 4(b) show the significant efficiency (small errors and small costs) of using reduced basis ANOVA for estimating the mean 
basis) Monte Carlo methods. As discussed before, the standard ANOVA in our test problem settings may not be more efficient than Monte Carlo methods when estimating the variance functions. Here it can be seen that the reduced basis ANOVA is very efficient for estimating variance functions. Figure 4(c) and Figure 4(d) show that, to achieve the same accuracy in variance estimates obtained by the standard Monte Carlo with around $10^{4}$ samples (similarly, that obtained by the standard ANOVA with order $i=2$ ), the reduced basis ANOVA with different residual error tolerances still only requires around ten percent of the costs of the Monte Carlo methods (or the standard ANOVA method).

The CPU times of ANOVA and Monte Carlo methods are presented in Table 4. It is clear that the reduced basis ANOVA method (denoted by RBA $\left(t_{o l} l_{r b}\right)$ associated with different values of the residual error tolerance: $t_{r l} l_{r b}=10^{-4}, 10^{-5}, 10^{-6}$ ) is much faster than the standard ANOVA method. Since the CPU times of the standard Monte Carlo method is very similar to that of the standard ANOVA method, it is not presented here. In addition, the direct reduced basis Monte Carlo method can be less efficient than standard ANOVA (or standard Monte Carlo) when comparing CPU times. This is not surprising. Since the estimated ranks of the full snapshot sets of these test problems are large (see Section 5.3), the direct reduced basis Monte Carlo method leads to large dense linear systems, and solving them can then be more expensive than solving sparse linear systems arising from the original finite element approximation. In addition, it is clear that small computational cost (or CPU times) of the reduced basis ANOVA method is due to the small number of full system (17) solves, i.e., the reduced basis sizes are small. Small reduced basis sizes also lead to small memory storage spentonly the reduced bases and the corresponding coefficients to represent each reduced solution needs to be stored for the reduced basis ANOVA, while the standard ANOVA method needs to store snapshots at all collocation points for each ANOVA term. When saving Monte Carlo solution samples, the memory storage required the direct reduced basis Monte Carlo is not significantly smaller than that of the standard Monte Carlo (or the standard ANOVA) for these test problems, since the direct reduced basis Monte 495 Carlo method requires large reduced basis sizes for these test problems (see Section 5.3. 

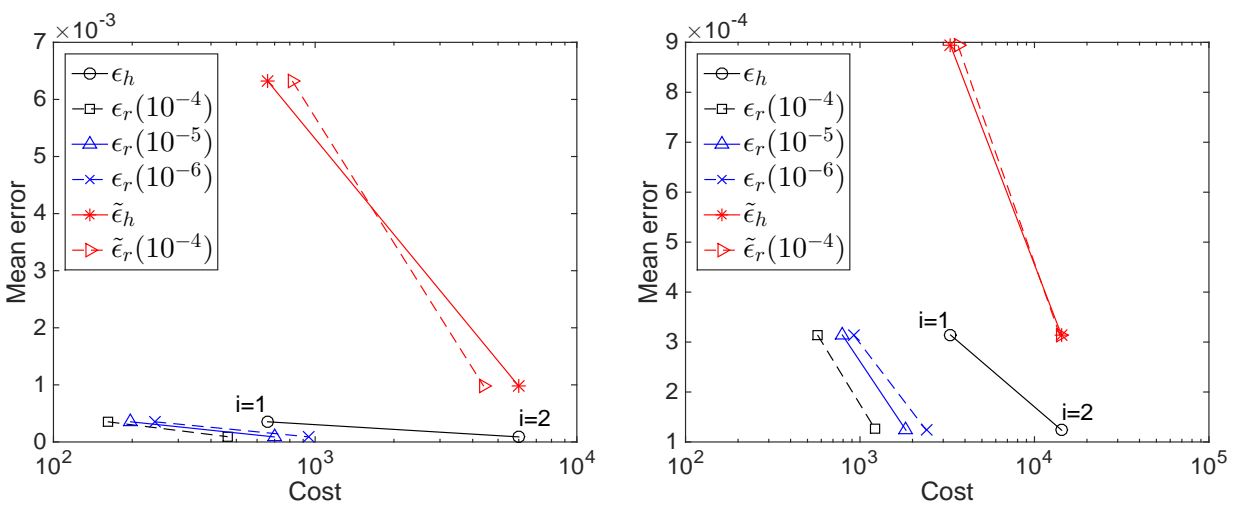

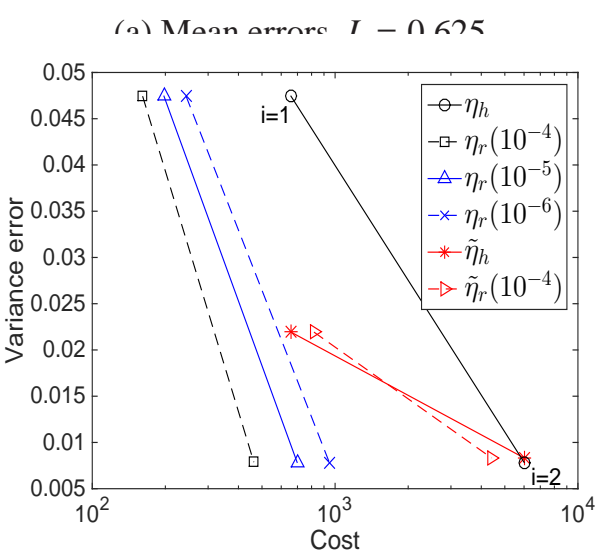

(c) Variance errors, $L=0.625$

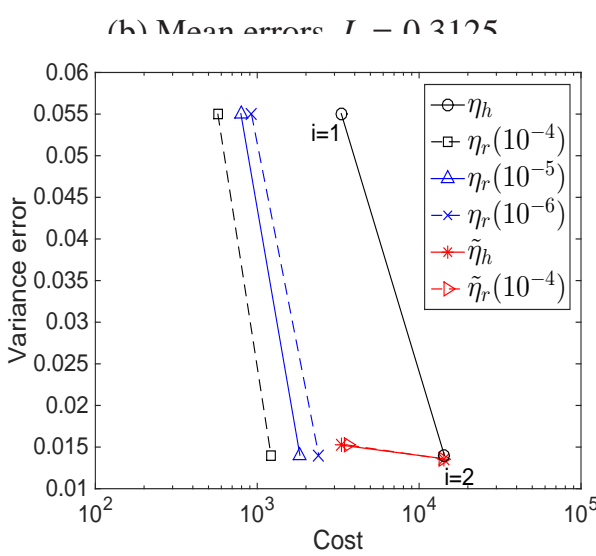

(d) Variance errors, $L=0.3125$

Figure 4: Errors in mean and variance function estimates of the standard ANOVA ( $\epsilon_{h}$ and $\left.\eta_{h}\right)$ and errors of the reduced basis ANOVA $\left(\epsilon_{r}\left(t o l_{r b}\right)\right.$ and $\eta_{r}\left(t o l_{r b}\right)$ associated with different values of the residual error tolerance: $\left.t l_{r b}=10^{-4}, 10^{-5}, 10^{-6}\right)$, are compared with errors of the standard Monte Carlo $\left(\tilde{\epsilon}_{h}\right.$ and $\left.\tilde{\eta}_{h}\right)$ and errors of the reduced basis Monte Carlo $\left(\tilde{\epsilon}_{r}\left(10^{-4}\right)\right.$ and $\left.\tilde{\eta}_{r}\left(10^{-4}\right)\right)$. 
Table 4: CPU times in seconds of the standard ANOVA (ANOVA), the reduced basis ANOVA (RBA(tol $\left.l_{r b}\right)$ associated with different values of the residual error tolerance: $t_{r} l_{r b}=10^{-4}, 10^{-5}, 10^{-6}$ ), and the reduced basis Monte Carlo with residual error tolerance $t_{l} l_{r b}=10^{(-4)}$ (denoted by $\operatorname{RMC}\left(10^{(-4)}\right)$ ).

\begin{tabular}{|c|c|c|c|c|c|}
\hline \multicolumn{6}{|c|}{ Results for $L=0.625$} \\
\hline Method & ANOVA & $\operatorname{RBA}\left(10^{-4}\right)$ & $\operatorname{RBA}\left(10^{-5}\right)$ & $\operatorname{RBA}\left(10^{-6}\right)$ & $\operatorname{RMC}\left(10^{-4}\right)$ \\
\hline CPU time & $7.25 e+00$ & $6.59 e-01$ & $9.70 e-01$ & $1.29 e+00$ & $3.82 e+01$ \\
\hline \multicolumn{6}{|c|}{ Results for $L=0.3125$} \\
\hline Method & ANOVA & $\operatorname{RBA}\left(10^{-4}\right)$ & $\operatorname{RBA}\left(10^{-5}\right)$ & $\operatorname{RBA}\left(10^{-6}\right)$ & $\operatorname{RMC}\left(10^{-4}\right)$ \\
\hline CPU time & $1.76 e+01$ & $2.08 e+00$ & $3.03 e+00$ & $3.53 e+00$ & $2.46 e+02$ \\
\hline
\end{tabular}

\subsection{Numerical study for the Stokes equations}

We next consider the Stokes equations with uncertain viscosity $a=a(x, \xi)$,

$$
\begin{array}{rll}
-a \nabla^{2} u+\nabla p=0 & \text { in } & D \times \Gamma, \\
\nabla \cdot u=0 & \text { in } & D \times \Gamma, \\
u=g & \text { on } & \partial D \times \Gamma,
\end{array}
$$

where $u=\left[u_{1}, u_{2}\right]^{T}$ is the flow velocity, $p$ is the scalar pressure. This kind of stochastic Stokes equations is also studied in $[66,6$, 67]. With the standard function space notation $\boldsymbol{H}^{1}:=H^{1}(D)^{2}, \boldsymbol{H}_{E}^{1}:=\left\{u \in \boldsymbol{H}^{1} \mid u=g\right.$ on $\left.\partial D\right\}, \boldsymbol{H}_{0}^{1}:=\left\{u \in \boldsymbol{H}^{1} \mid u=\right.$ $[0,0]^{T}$ on $\left.\partial D\right\}$, and $L_{0}^{2}(\Omega):=\left\{q \in L^{2}(D) \mid \int_{D} q d D=0\right\}$, the weak form of the deterministic problem associated with (38)-(40) is: find $u \in \boldsymbol{H}_{E}^{1}$ and $p \in L_{0}^{2}(D)$, such that

$$
\begin{aligned}
(a \nabla u, \nabla v)-(p, \nabla \cdot v) & =0 \quad \forall v \in \boldsymbol{H}_{0}^{1}, \\
(\nabla \cdot u, q) & =0 \quad \forall q \in L_{0}^{2}(D) .
\end{aligned}
$$

The Dirichlet flow boundary condition condition (40) can be non-homogeneous. For simplicity, we can take $u=\tilde{u}+u^{b c}$ where $\tilde{u}$ denotes the interior part and $u^{b c}$ denotes the boundary part of the solution (see [45] for detailed discussions), and reformulate 
(41)-(42) as: find $\tilde{u} \in \boldsymbol{H}_{0}^{1}$ and $p \in L_{0}^{2}(D)$, such that

$$
\begin{aligned}
(a \nabla \tilde{u}, \nabla v)-\left(p^{0}, \nabla \cdot v\right) & =-\left(\nabla u^{b c}, \nabla v\right) \quad \forall v \in \boldsymbol{H}_{0}^{1}, \\
(\nabla \cdot \tilde{u}, q) & =-\left(\nabla \cdot u^{b c}, q\right) \quad \forall q \in L_{0}^{2}(D) .
\end{aligned}
$$

Mixed finite element approximation of (43)-(44) is obtained by choosing finite dimensional subspaces $X_{0}^{h}$ and $M^{h}$ of $\boldsymbol{H}_{0}^{1}$ and $\boldsymbol{H}_{0}^{1}$ and $M^{h}$ respectively [58]. The finite element solutions (snapshots) are denoted $u_{h}$ and $p_{h}$. Moreover, reduced basis approximation is obtained by introducing reduced bases $U \subset X_{0}^{h}$ and $P \subset M^{h}$, and reduced basis approximations for velocity and pressure solutions are denoted by $u_{r}$ and $p_{r}$ respectively. The pair of reduced bases $U$ and $P$ must be defined properly to satisfy the inf-sup condition. As introduced in [68】, one way to restore the inf-sup stability is to construct the velocity reduced basis from two parts- $U:=\tilde{U} \cup \hat{U}$ where $\tilde{U}$ is obtained form velocity snapshots and $\hat{U}$ is an additional function set to restore the stability. The set $\hat{U}$ is constructed as follows [68]: for each $p \in P$, we find its corresponding stability-enrichment function $u^{p} \in X_{0}^{h}$ satisfying

$$
\left(\nabla u^{p}, \nabla v\right)=(p, \nabla \cdot v) \quad \forall v \in X_{0}^{h},
$$
$\sigma=0.5$ and covariance function (24). We again set the correlation length $L=0.625$, and take $M=73$ in (25) to capture $95 \%$ of the total variance, and set the random variables $\left\{\xi_{k}\right\}_{k=1}^{M}$ in (25) to be independent uniform distributions with range $I=[-1,1]$. 
For this driven cavity test problem, we apply our reduced basis ANOVA algorithm (Algorithm 2) to generate the ANOVA-collocation approximation for both velocity and pressure solutions. Implementation details of using Algorithm 2 to solve the Stokes equations with mixed finite elements are presented as follows.

The first step here is the same as that in Section 4, We set $\mathcal{J}_{0}:=\{\emptyset\}$, compute the velocity snapshot $u_{h}(x, c)$ and the pressure snapshot $p_{h}(x, c)$, and set the zeroth ANOVA order terms $u_{\emptyset}^{\mathrm{sc}}\left(x, \xi_{\emptyset}\right):=u_{h}(x, c)$ and $u_{\emptyset}^{\mathrm{sc}}\left(x, \xi_{\emptyset}\right):=p_{h}(x, c)$. We construct $\tilde{U}_{\emptyset}:=$ $\left\{u_{h}(x, c)\right\}$ and $P_{\emptyset}:=\left\{p_{h}(x, c)\right\}$ (where the subscript $\emptyset$ is the same as that introduced in Section 2.11, and define a first order index set by $\mathcal{J}_{1}:=\{1, \ldots, M\}$.

Second (see Section 44), given an ANOVA order $i \geq 1$, supposing the index set $\mathcal{J}_{i}$ and the reduced bases for the previous order $\left(\tilde{U}_{s}\right.$ and $P_{s}$ for all $s \in \mathcal{J}_{i-1}$ with the subscript notation introduced in Section (4) are given, the initialize reduced basis $U_{t}$ and $P_{t}$ for $t \in \mathcal{J}_{i}$ are constructed as follows:

1. define $\tilde{U}_{t}^{0}:=\cup_{s \in \Lambda_{t}} \tilde{U}_{s}^{0}$ and $P_{t}^{0}:=\cup_{s \in \Lambda_{t}} P_{s}$ where $\Lambda_{t}:=\left\{s \mid s \in \mathfrak{I}_{|t|-1}\right.$ and $\left.s \subset t\right\}$;

2. initially set $\tilde{U}_{t}:=\operatorname{POD}\left(\tilde{U}_{t}^{0}\right)$ and $P_{t}:=\operatorname{POD}\left(P_{t}^{0}\right)$ (details of POD are discussed in Section 3 ;

3. for each pressure reduced basis function $p \in P_{t}$, we compute its corresponding stability-enrichment function $u^{p}$ through solving (45), and set $\hat{U}_{t}:=\left\{u_{p}, \forall p \in\right.$ $\left.P_{t}\right\}$ and $Q_{t}:=\tilde{U}_{t} \cup \hat{U}_{t}$.

The other parts of the implementation details of the reduced basis ANOVA algorithm are the same with that in Section 4 , except for the following two additional modifications. When updating the reduced bases during the greedy procedure over the sparse grids, we need to update $\tilde{U}_{t}$ and $P_{t}$ through new snapshots, while we also need to update $\hat{U}_{t}$ with the function obtained from solving (45) (the overall velocity reduced basis is $Q_{t}:=\tilde{U}_{t} \cup \hat{U}_{t}$ ). In addition, we denote terms of the ANOVA-collocation approximation for velocity and pressure solutions by $u_{t}^{\mathrm{sc}}$ and $p_{t}^{\mathrm{sc}}$ respectively (see (13)-(14)), and define relative mean value for the Stokes problem by

$$
\tilde{\gamma}_{t}:=\frac{\left\|\tilde{\mathbf{E}}\left(u_{t}^{\mathrm{sc}}\right)\right\|_{0, D}+\left\|\tilde{\mathbf{E}}\left(p_{t}^{\mathrm{sc}}\right)\right\|_{0, D}}{\left\|\sum_{s \in \mathcal{J}_{|| \mid-1}^{\star}} \tilde{\mathbf{E}}\left(u_{s}^{\mathrm{sc}}\right)\right\|_{0, D}+\left\|\sum_{s \in \mathcal{J}_{|| \mid-1}^{\star}} \tilde{\mathbf{E}}\left(p_{s}^{\mathrm{sc}}\right)\right\|_{0, D}} .
$$


To generate reference results, we use the standard Monte Carlo method with $10^{6}$ samples (using the mixed finite element method to solve (41)-(42) to generate solution samples). In Table 5, three methods are compared: MC refers to the standard Monte Carlo method; ANOVA refers to the standard ANOVA method; RBA $\left(t_{o l} l_{r b}\right)$ refers to the reduced basis ANOVA method with different residual error tolerance values (see line 11 of Algorithm 2), while the tolerance for the relative mean value is set to tol ${ }_{\text {anova }}=10^{-4}$ (see line 22 of Algorithm 2) in all our numerical studies. The quantities in Table 5 are defined in the same way as those for the diffusion test problems: the CPU time refers to the sum of CPU times of linear system solves involved in each method; relative mean and variance errors are defined as in the same way as (30)-(35) for Monte Carlo and ANOVA methods (we here add velocity and pressure errors together).

From Table 5 compared with the standard ANOVA method, the reduced basis ANOVA method has significantly smaller CPU times, while the standard and the reduced basis ANOVA methods have similar mean and variance errors (for both velocity and pressure approximation). Comparing the standard ANOVA with the standard Monte Carlo (we use 1144 samples for the standard Monte Carlo such that its CPU time is similar to that of the standard ANOVA), errors of the standard ANOVA method are clearly smaller than those of the standard Monte Carlo method.

Table 5: CUP time in seconds, errors in mean and variance function estimates of the standard Monte Carlo (MC), the standard ANOVA (ANOVA), the reduced basis ANOVA $\left(\operatorname{RBA}\left(t_{o l} l_{r b}\right)\right.$ associated with different values of the residual error tolerance: $t_{r b}=10^{-4}, 10^{-5}, 10^{-6}$ ).

\begin{tabular}{lccc}
\hline Method & CPU time & mean error & variance error \\
\hline MC & $1.02 e+02$ & $4.76 e-03$ & $8.22 e-02$ \\
ANOVA & $1.01 e+02$ & $1.55 e-04$ & $3.73 e-02$ \\
$\operatorname{RBA}\left(10^{-4}\right)$ & $7.99 e+00$ & $3.26 e-04$ & $3.68 e-02$ \\
$\operatorname{RBA}\left(10^{-5}\right)$ & $1.40 e+01$ & $1.57 e-04$ & $3.74 e-02$ \\
$\operatorname{RBA}\left(10^{-6}\right)$ & $1.90 e+01$ & $1.56 e-04$ & $3.74 e-02$ \\
\hline
\end{tabular}




\section{Summary and conclusions}

This paper describes the mathematical framework and implementation of the reduced basis ANOVA method for solving partial differential equations with high-dimensional random inputs. We consider the nested structures of ANOVA indices and build reduced bases hierarchically to identify the low-rank structures in the collocation coefficients associated with each ANOVA expansion term. Numerical studies demonstrate that this new approach can significantly improve the computational efficiency of the standard ANOVA-collocation approach without compromising accuracy.

The performance of the proposed reduced basis ANOVA method for solving partial differential equations with high-dimensional random inputs depend on the structure of random inputs. It is well-known that the standard ANOVA method is efficient when the random inputs have additive structures, while it may not be so efficient when the random inputs are non-additive. Solving problems with non-additive random inputs is therefore a main bottleneck for our method. Overcoming this bottleneck is a grand challenge and we will address it in our future research. In addition, reduced basis methods have well-known limitations for problems that are truly high-dimensional. Effective dimension reduction algorithms will be investigated for such problems. Although adaptive ANOVA can effectively solve for problems with high-dimensional inputs and high-variability within a desired accuracy, the adaptivity criteria for ANOVA decompositions are mostly heuristic. We will investigate more mathematical rigorous adaptive criteria in our future work so that no important ANOVA terms will be ignored. Finally, the choice of anchor points can result in different performances of the anchored ANOVA expansions. In this paper the mean value of the inputs is served as the anchor point, while developing systematical approaches to choose accurate anchor points for the proposed reduced basis ANOVA method will be investigated in our future work.

Acknowledgments: G. Lin would like to acknowledge the support by NSF Grant DMS-1555072, and by the U.S. Department of Energy, Office of Science, Office of Advanced Scientific Computing Research, Applied Mathematics program as part of the Multifaceted Mathematics for Complex Energy Systems ( $\left.\mathrm{M}^{2} \mathrm{ACS}\right)$ project and part of the Collaboratory on Mathematics for Mesoscopic Modeling of Materials project. 


\section{Reference}

${ }_{610}$ [1] D. Xiu, G. Karniadakis, The Wiener-Askey polynomial chaos for stochastic differential equations, SIAM Journal on Scientific Computing 24 (2002) 619-644.

[2] R. Ghanem, P. Spanos, Stochastic Finite Elements: A Spectral Approach, Dover Publications, New York, 2003.

[3] D. Xiu, J. Hesthaven, High-order collocation methods for differential equations with random inputs, SIAM Journal on Scientific Computing 27 (2005) 11181139.

[4] F. Nobile, R. Tempone, C. G. Webster, A sparse grid stochastic collocation method for partial differential equations with random input data, SIAM Journal on Numerical Analysis 46 (2008) 2309-2345.

[5] D. Xiu, Numerical Methods for Stochastic Computations: A Spectral Method Approach, Princeton University Press, Princeton, 2010.

[6] H. Elman, Q. Liao, Reduced basis collocation methods for partial differential equations with random coefficients, SIAM/ASA Journal on Uncertainty Quantification 1 (2013) 192-217.

[7] R. Fisher, Statistical Methods for Research Workers, Oliver and Boyd, Berlin, 1925.

[8] Y. Cao, Z. Chen, M. Gunzburger, Anova expansions and efficient sampling methods for parameter dependent nonlinear PDEs, International Journal of Numerical Analysis and Modeling 6 (2009) 256-273.

[9] C. Winter, A. Guadagnini, D. Nychka, D. Tartakovsky, Multivariate sensitivity analysis of saturated flow through simulated highly heterogeneous groundwater aquifers, Journal of Computational Physics 217 (2009) 166-175.

[10] J. Foo, G. Karniadakis, Multi-element probabilistic collocation in high dimensions, Journal of Computational Physics 229 (2010) 1536-1557. 
635

[11] Z. Gao, J. S. Hesthaven, On anova expansions and strategies for choosing the anchor point, Applied Mathematics and Computation 217 (2010) 3274-3285.

[12] X. Ma, N. Zabaras, An adaptive high-dimensional stochastic model representation technique for the solution of stochastic partial differential equations, Journal of Computational Physics 229 (2010) 3884-3915.

[13] Z. Zhang, M. Choi, G. Karniadakis, Anchor points matter in anova decomposition, Spectral and High Order Methods for Partial Diferential Equations Lecture Notes in Computational Science and Engineering 76 (2011) 347-355.

[14] X. Yang, M. Choi, G. Lin, G. E. Karniadakis, Adaptive anova decomposition of stochastic incompressible and compressible flows, Journal of Computational Physics 231 (2012) 1587-1614.

[15] J. S. Hesthaven, S. Zhang, On the use of anova expansions in reduced basis methods for high-dimensional parametric partial differential equations, Journal of Scientific Computing, To appear, DOI: 10.1007/s10915-016-0194-9.

[16] H. J. Bungartz, M. Griebel, Sparse grids, Acta Numerica 13 (2004) 147-269.

[17] B. Ganapathysubramanian, N. Zabaras, Sparse grid collocation schemes for stochastic natural convection problems, Journal of Computational Physics 225 (2007) 652-685.

[18] D. Xiu, Efficient collocational approach for parametric uncertainty analysis, Communications in Computational Physics 2 (2007) 293-309.

[19] N. Agarwal, N. R. Aluru, A domain adaptive stochastic collocation approach for analysis of MEMS under uncertainties, Journal of Computational Physics 194 (2009) 7662-7688.

[20] X. Ma, N. Zabaras, An adaptive hierarchical sparse grid collocation algorithm for the solution of stochastic differential equations, Journal of Computational Physics 228 (2009) 3084-3113. 
[21] X. Wan, G. E. Karniadakis, An adaptive multi-element generalized polynomial chaos method for stochastic differential equations, Journal of Computational Physics 209 (2005) 617-642.

[22] A. Doostan, H. Owhadi, A non-adaptive sparse approximation for PDEs with stochastic inputs, Journal of Computational Physics 230 (2011) 3015-3034.

[23] L. Yan, L. Guo, D. Xiu, Stochastic collocation algorithms using L1-minimization, International Journal for Uncertainty Quantification 3 (2012) 279-293.

[24] G. Karagiannis, B. Konomi, G. Lin, Mixed shrinkage prior procedure for basis selection and global evaluation of gPC expansions in bayesian framework: Applications to elliptic SPDEs, Journal of Computational Physics 284 (2015) 528-546.

[25] J. Eftang, A. Patera, E. Rønquist, An "hp" certified reduced basis method for parametrized elliptic partial differential equations, SIAM Journal on Scientific Computing 32 (2010) 3170-3200.

[26] B. Peherstorfer, D. Butnaru, K. Willcox, H. Bungartz, Localized discrete empirical interpolation method, SIAM Journal on Scientific Computing 36 (2014) A168-A192.

[27] F. Albrecht, B. Haasdonk, M. Ohlberger, S. Kaulmann, The localized reduced basis multiscale method, in: Proceedings of Algoritmy 2012, Conference on Scientific Computing, Slovak University of Technology in Bratislava, Publishing House of STU, Vysoke Tatry, Podbanske, 2012, pp. 393-403.

[28] D. Huynh, D. Knezevic, A. Patera, A static condensation reduced basis element method : approximation and a posteriori error estimation, ESAIM: Mathematical Modelling and Numerical Analysis 47 (2013) 213-251.

[29] I. Maier, B. Haasdonk, A Dirichlet-Neumann reduced basis method for homogeneous domain decomposition problems, Applied Numerical Mathematics 78 (2014) 31-48. 
[30] A. Sarkar, N. Benabbou, R. Ghanem, Domain decomposition of stochastic PDEs: Theoretical formulations, International Journal for Numerical Methods in Engineering 77 (2009) 689-701.

[31] M. Hadigol, A. Doostan, H. G. Matthies, R. Niekamp, Partitioned treatment of uncertainty in coupled domain problems: A separated representation approach, Computer Methods in Applied Mechanics and Engineering 274 (2014) 103-124.

[32] Y. Chen, J. Jakeman, C. Gittelson, D. Xiu, Local polynomial chaos expansion for linear differential equations with high dimensional random inputs, SIAM Journal on Scientific Computing 37 (2015) A79-A102.

[33] F. J. Alexander, A. L. Garcia, D. M. Tartakovsky, Algorithm refinement for stochastic partial differential equations: II. Correlated systems, Journal of Computational Physics 207 (2005) 769-787.

[34] M. Arnst, R. Ghanem, E. Phipps, J. Red-Horse, Dimension reduction in stochastic modeling of coupled problems, International Journal for Numerical Methods in Engineering 92 (2012) 940-968.

[35] S. Amaral, D. Allaire, K. Willcox, A decomposition-based approach to uncertainty analysis of feed-forward multicomponent systems, International Journal for Numerical Methods in Engineering 100 (2014) 982-1005.

[36] Q. Liao, K. Willcox, A domain decomposition approach for uncertainty analysis, SIAM Journal on Scientific Computing 37 (2015) A103-A133.

[37] B. Ganapathysubramanian, N. Zabaras, Modeling diffusion in random heterogeneous media: Data-driven models, stochastic collocation and the variational multiscale method, Journal of Computational Physics 226 (2007) 326-353.

[38] P. Chen, A. Quarteroni, G. Rozza, Comparison between reduced basis and stochastic collocation methods for elliptic problems, Journal of Scientific Computing 59 (2014) 187-216. 
[39] P. Chen, A. Quarteroni, A new algorithm for high-dimensional uncertainty quantification based on dimension-adaptive sparse grid approximation and reduced basis methods, Journal of Computational Physics 298 (2015) 176-193.

[40] I. Sobol, Theorems and examples on high dimensional model representation, Reliability Engineering and System Safety 79 (2003) 187-193.

[41] H. Elman, V. Forstall, Preconditioning techniques for reduced basis methods for parameterized elliptic partial differential equations, SIAM Journal on Scientific Computing 37 (2015) S177-S194.

[42] P. Benner, S. Gugercin, K. Willcox, A survey of model reduction methods for parametric systems, SIAM Review to appear.

[43] L. Sirovich, Turbulence and the dynamics of coherent structures, Part I: Coherent structures, Quarterly of Applied Mathematics 45 (1987) 561-571.

[44] P. Holmes, J. L. Lumley, G. Berkooz, Turbulence, Coherent Structures, Dynamical Systems and Symmetry, Cambridge, New York, 1996.

[45] M. Gunzburger, J. Peterson, J. Shadid, Reduced-order modeling of timedependent PDEs with multiple parameters in the boundary data, Computer Methods in Applied Mechanics and Engineering 196 (2007) 1030-1047.

[46] K. Veroy, D. Rovas, A. Patera, A posteriori error estimation for reducedbasis approximation of parametrized elliptic coercive partial differential equations:"Convex Inverse" bound conditioners, ESAIM: Control, Optimisation and Calculus of Variations 8 (2002) 1007-1028.

[47] N. Nguyen, K. Veroy, A. Patera, Certified real-time solution of parametrized partial differential equations, in: S. Yip (Ed.), Handbook of Materials Modeling, Springer, 2005, pp. 1523-1558.

[48] B. Haasdonk, M. Ohlberger, Reduced basis method for finite volume approximations of parametrized linear evolution equations, ESAIM: Mathematical Modelling and Numerical Analysis 42 (2008) 277-302. 
[49] S. Boyaval, C. L. Bris, T. Lelièvre, Y. Maday, N. Nguyen, A. Patera, Reduced basis techniques for stochastic problems, Archives of Computational Methods in Engineering 17 (2010) 1-20.

[50] A. Patera, G. Rozza, Reduced Basis Approximation and A Posteriori Error Estimation for Parametrized Partial Differential Equations, 2007, version 1.0, Copyright MIT 2006-2007, to appear in (tentative title) MIT Pappalardo Graduate Monographs in Mechanical Engineering.

[51] A. Quarteroni, A. Manzoni, F. Negri, Reduced Basis Methods for Partial Differential Equations, Springer International Publishing, Springer International Publishing Switzerland, 2016.

[52] T. Bui-Thanh, K. Willcox, O. Ghattas, Model reduction for large-scale systems with high-dimensional parametric input space, SIAM Journal on Scientific Computing 30 (2008) 3270-3288.

[53] G. Rozza, D. Huynh, A. Patera, Reduced basis approximation and a posteriori error estimation for affinely parametrized elliptic coercive partial differential equations: Application to transport and continuum mechanics, Archives of Computational Methods in Engineering 15 (2008) 229-275.

[54] M. Grepl, Y. Maday, N. Nguyen, A. Patera, Efficient reduced-basis treatment of nonaffine and nonlinear partial differential equations, Mathematical Modelling and Numerical Analysis 41 (2007) 575-605.

[55] S. Chaturantabut, D. Sorensen, Nonlinear model reduction via discrete empirical interpolation, SIAM Journal on Scientific Computing 32 (2010) 2737-2764.

[56] H. Elman, C. Miller, E. Phipps, R. Tuminaro, Assessment of collocation and Galerkin approaches to linear diffusion equations with random data, International Journal for Uncertainty Quantification 1 (2011) 19-34.

[57] D. Braess, Finite Elements, Cambridge University Press, London, 1997. 
[58] H. Elman, D. Silvester, A. Wathen, Finite Elements and Fast Iterative Solvers, Oxford University Press, New York, 2005.

[59] I. Babuška, F. Nobile, R. Tempone, A stochastic collocation method for elliptic partial differential equations with random input data, SIAM Journal on Numerical Analysis 45 (2007) 1005-1034.

[60] J. L. Brown, Jr., Mean square truncation error in series expansions of random functions, Journal of the Society for Industrial and Applied Mathematics 8 (1960) $28-32$.

[61] C. Schwab, R. A. Todor, Karhunen-Loève approximation of random fields by generalized fast multipole methods, J. Comput. Phys. 217 (2006) 100-122.

[62] A. Klimke, Sparse Grid Interpolation Toolbox - user's guide, Tech. Rep. IANS report 2007/017, University of Stuttgart (2007).

[63] K. Tang, P. M. Congedo, R. Abgrall, Sensitivity analysis using anchored ANOVA expansion and high-order moments computation, International Journal for $\mathrm{Nu}-$ merical Methods in Engineering 102 (2015) 1554-1584.

[64] L. Ng, K. Willcox, Multifidelity approaches for optimization under uncertainty, International Journal for Numerical Methods in Engineering 100 (2014) 746-772.

[65] X. Zhu, A. Narayan, D. Xiu, Computational aspects of stochastic collocation with multifidelity models, SIAM/ASA Journal on Uncertainty Quantification 2 (2014) $444-463$.

[66] C. Powell, D. Silvester, Preconditioning steady-state navier-stokes equations with random data, SIAM Journal on Scientific Computing 34 (2012) A2482-A2506.

[67] P. Chen, A. Quarteroni, G. Rozza, Multilevel and weighted reduced basis method for stochastic optimal control problems constrained by stokes equations, $\mathrm{Nu}-$ merische Mathematik (2015) 1-36. 
[68] A. Quarteroni, G. Rozza, Numerical solution of parametrized Navier-Stokes equations by reduced basis methods, Numerical Methods for Partial Differential Equations 23 (2007) 923-948. 